\title{
Types of the canonical isometric imbeddings of symmetric $R$-spaces
}

\author{
Dedicated to Professor Noboru Tanaka on his 60th birthday
}

Eiji KANEDA

(Received February 20, 1992, Revised May 8, 1992)

\section{Introduction.}

In the history of Riemannian geometry, the rigidity problem of isometric imbeddings has been one of the major problems and has been studied by many authors.

Let $\boldsymbol{f}$ be an isometric imbedding of a Riemannian manifold $M$ into a euclidean space $\boldsymbol{R}^{m}$. $\boldsymbol{f}$ is called rigid if any other isometric imbedding of $M$ into $\boldsymbol{R}^{m}$ can be written by a composite of $\boldsymbol{f}$ and a euclidean transformation of $\boldsymbol{R}^{m}$. The rigidity problem is to determine whether a given isometric imbedding $\boldsymbol{f}$ is rigid or not. In his paper [T/n], N. Tanaka threw a new light upon the rigidity problem and made a great contribution to the progress of this problem.

Let $\boldsymbol{f}$ be an isometric imbedding of $M$ into $\boldsymbol{R}^{m}$. We define a differential operator $\Phi_{* f}$ by setting

$$
\Phi_{* f}(\boldsymbol{u})=<\mathrm{d} \boldsymbol{f}, \mathrm{d} \boldsymbol{u}>,
$$

where $\boldsymbol{u}$ is a differentiable map of $M$ to $\boldsymbol{R}^{m}$. A solution $\boldsymbol{u}$ of the equation $\Phi_{* f}(\boldsymbol{u})=0$ is called an infinitesimal isometric deformation of $\boldsymbol{f}$. N. Tanaka proved that under the assumption that $\boldsymbol{f}$ is non-degenerate, there is a differential operator $L$ associated with $\boldsymbol{f}$, which is, in a sense, equivalent to the operator $\Phi_{* f}$ and the solution space of the equation $L \varphi=0$ is isomorphic with the space of infinitesimal isometric deformations of $\boldsymbol{f}$.

It is noted that the operator $L$ has a preferable property as a differential operator; the symbol of $L$ is not necessarily degenerate, although the symbol of $\Phi_{* f}$ is necessarily degenerate. Therefore, through the operator $L$, the rigidity problem can be observed from a viewpoint of the differential equation.

In [Tn], N. Tanaka studied the case where the operator $L$ is of elliptic type and $\boldsymbol{f}$ is infinitesimally rigid; an isometric imbedding $\boldsymbol{f}$ is called infinitesimally rigid if each solution of $L \varphi=0$ corresponds to an infinitesimal euclidean transformation of $\boldsymbol{R}^{m}$. Applying the theory of elliptic differential equation, he established a global rigidity theorem for such 
an isometric imbedding $\boldsymbol{f}$. As an application of this theorem, he proved actual rigidity theorems for the so called canonical isometric imbeddings of Hermitian symmetric spaces of compact type.

Studies in this line can be seen in [KT] and [Ka]; for the canonical imbeddings of real Grassmann manifolds $G^{2, q}(\boldsymbol{R})(q \geqq 3)$ and the classical compact Lie groups $U(n), S O(n)$ and $S p(n)$, the dimensions of the solution spaces of $L \varphi=0$ are calculated and actual global rigidity theorems have been established.

Kaneda and Tanaka [KT] studied the local solutions of the equation $L \varphi=0$; especially, the prolongations of the symbol of $L$ are systematically discussed. They considered the case where $L$ is of finite type, i. e., the equation $L \varphi=0$ reduces to a completely integrable system of differential equations after finitely many prolongation procedures. In case the symbol of $L$ has minimal prolongations, they proved that $f$ is locally rigid, i. e., even the restriction of $\boldsymbol{f}$ to any small open submanifold of $M$ is rigid in a sense. This local rigidity theorem is a refinement of the global rigidity theorem stated above; for many irreducible Hermitian symmetric spaces of compact type the actual global rigidity theorems can be also proved by this theorem.

In this paper, we say that a non-degenerate isometric imbedding $f$ is of type $(E)$ (resp. type $(F)$ ) if $L$ is of elliptic type (resp. finite type). As we have seen, the operator $L$ played an important role in the study of the global or local rigidity theorem; therefore, to discuss the rigidity of a given isometric imbedding $\boldsymbol{f}$ it is a fundamental and interesting problem to determine the type of $\boldsymbol{f}$.

The purpose of this paper is to survey the types of $L$ associated with the canonical isometric imbeddings of symmetric $R$-spaces $G / K$. Symmetric $R$-spaces form a wide class of compact Riemannian symmetric spaces; the irreducible Hermitian symmetric spaces of compact type, the real quadrics $Q^{p, q}(\boldsymbol{R})$ and the Grassmann manifolds $G^{p, q}(\boldsymbol{K})(\boldsymbol{K}=\boldsymbol{R}, \boldsymbol{C}$ or $\boldsymbol{H}$ ) belong to this class (see Table I Appendix 1). It is known that for each symmetric $R$-space $G / K$ there is an isometric imbedding into a euclidean space, which is called the canonical isometric imbedding of $G / K$

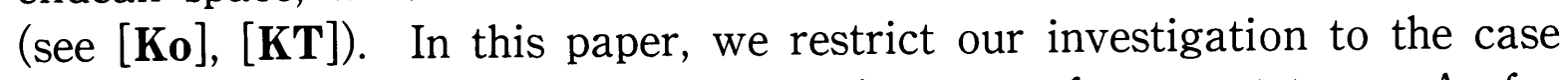
where $G / K$ is not a Hermitian symmetric space of compact type. As for the Hermitian symmetric spaces of compact type, the types of the canonical isometric imbeddings have been already known (see [Th], [KT]).

Roughly speaking, it is shown that for almost all symmetric $R$-spaces the canonical isometric imbeddings $\boldsymbol{f}$ are of type $(F)$ and that the exceptional cases are limited to the already known cases in [KT] (see Theorem 
2.6). We prove: (1) $\boldsymbol{f}$ is of type $(F)$ if $G / K$ does not coincide with any of the real quadrics $Q^{p, q}(\boldsymbol{R})(p, q \geqq 1)$, the real projective spaces $P^{n}(\boldsymbol{R})$ nor the real Grassmann manifolds $G^{2, q}(\boldsymbol{R})$; (2) $\boldsymbol{f}$ is of type $(E)$ if $G / K$ does not coincide with any of the real quadrics $Q^{p, q}(\boldsymbol{R})$ nor the real projective spaces $P^{n}(\boldsymbol{R})$. Accordingly, for almost all symmetric $R$-spaces, we can conclude that the space of (local) infinitesimal isometric deformations of $\boldsymbol{f}$ is of finite dimension.

Throughout this paper we assume the differentiability of class $C^{\infty}$. Unless otherwise stated, for Lie groups, Lie algebras and symmetric spaces, we follow the notation in $[\mathbf{H}]$.

\section{$\S 1$. Types of isometric imbeddings.}

1. 1. The differential operator $L$. Let $(M, g)$ be an $n$-dimensional Riemannian manifold. We denote by $T(M)$ (resp. $T^{*}(M)$ ) the tangent (resp. cotangent) bundle of $M$. We also denote by $S^{2} T^{*}(M)$ the symmetric product of $T^{*}(M)$.

Let $\boldsymbol{f}$ be an isometric imbedding of $(M, g)$ into a euclidean space $\boldsymbol{R}^{m}$. We denote by $N(M)$ the normal bundle of $\boldsymbol{f}$. Let $\nabla$ be the Riemannian connection of $(M, g)$. Then, it is known that at each $p \in M$, the second derivative $\nabla_{x} \nabla_{y} f, x, y \in T_{p}(M)$ takes its value in the normal vector space $N_{p}(M)$. Let $A \in N_{p}(M)$. We define a symmetric bilinear form $A^{\vee}$ by

$$
A^{\curlyvee}(x, y)=<A, \nabla_{x} \nabla_{y} \boldsymbol{f}>, x, y \in T_{p}(M) .
$$

$A^{\vee}$ is called the second fundamental form of $\boldsymbol{f}$ with respect to $A$. We say that an isometric imbedding $\boldsymbol{f}$ is non-degenerate if at each $p \in M, N_{p}(M)$ is spanned by the vectors $\nabla_{x} \nabla_{y} f\left(x, y \in T_{p}(M)\right)$.

Let $\boldsymbol{f}$ be a non-degenerate isometric imbedding of $(M, g)$ into $\boldsymbol{R}^{m}$. Then the map $N_{p}(M) \ni A \longmapsto A^{\vee} \in S^{2} T_{p}^{*}(M)$ is injective for each $p \in M$. We denote by $N_{p}^{\vee}$ the subspace of $S^{2} T_{p}^{*}(M)$ consisting of all $A^{\vee}(A \in$ $N_{p}(M)$ ). It is easy to see that $N^{\vee}=\cup_{p \in M} N_{p} \vee$ forms a subbundle of $S^{2} T^{*}(M)$, which is called the bundle of second fundamental forms associated with $\boldsymbol{f}$. We denote by $\pi$ the natural projection of $S^{2} T^{*}(M)$ onto the quotient bundle $S^{2} T^{*}(M) / N^{\vee}$.

We now recall the definition of the differential operator $L$. Let $\varphi$ be a differential 1-form on $M$. Define a symmetric 2-tensor field $D \varphi$ on $M$ by

$$
D \varphi(x, y)=\nabla_{x} \varphi(y)+\nabla_{y} \varphi(x), x, y \in T_{p}(M) .
$$

It is easily shown that $D \varphi=\mathscr{L}_{X} g$, where $X$ is the vector field on $M$ defined by $\varphi(\bullet)=g(X, \cdot)$ and $\mathscr{L}_{X} g$ is the Lie derivative of $g$ by $X$. The differential operator $L$ is then defined as a composite $\pi \cdot D$, i. e., 


$$
L: \Gamma\left(T^{*}(M)\right) \ni \varphi \longmapsto \pi(D \varphi) \in \Gamma\left(S^{2} T^{*}(M) /\left(N^{\vee}\right) .\right.
$$

The following proposition is fundamental in the discussion of infinitesimal isometric deformations of $\boldsymbol{f}$.

Proposition 1.1 (see [Tn], [KT]). Let $\boldsymbol{f}$ be an isometric imbedding of a Riemannian manifold $(M, g)$ into a euclidean space $\boldsymbol{R}^{m}$. Assume that $\boldsymbol{f}$ is non-degenerate. Then there exists a linear isomorphism between the kernel of the operator $L$ and the space of infinitesimal isometric deformations of $\boldsymbol{f}$.

1.2. Properties $(\boldsymbol{E})$ and $(\boldsymbol{F})$. Before proceeding to the definition of type of isometric imbeddings, we make a brief preparation for symmetric bilinear forms.

Let $V$ be an $n$-dimensional vector space over $\boldsymbol{R}$. We denote by $V^{*}$ the dual vector space of $V$ and by $S^{2} V^{*}$ the symmetric product of $V^{*}$. We also denote by $V^{* c}$ and $S^{2} V^{* c}$ the complexifications of $V^{*}$ and $S^{2} V^{*}$.

Let $A$ be an element of $S^{2} V^{*}$. $A$ is called decomposable if it can be expressed as a symmetric product of two vectors in $V^{*}$, i. e., $A=\xi_{1} \bullet \xi_{2}, \xi_{1}$, $\xi_{2} \in V^{*}$. Similarly, an element $B \in S^{2} V^{* c}$ is called decomposable if it can be expressed as a symmetric product of two vectors in $V^{* c}$, i. e., $B=\eta_{1} \bullet \eta_{2}$, $\eta_{1}, \eta_{2} \in V^{* c}$.

Definition. Let $W$ be subspace of $S^{2} V^{*}$. We say that $W$ has Property $(E)$ if $W$ contains no non-trivial decomposable elements of $S^{2} V^{*}$. We also say that $W$ has Property $(F)$ if the complexification $W^{c}$ of $W$ contains no non-trivial decomposable elements of $S^{2} V^{* c}$.

DEFINITION. Let $\boldsymbol{f}$ be a non-degenerate isometric imbedding of $(M$, $g$ ) into a euclidean space $\boldsymbol{R}^{m}$. Then $\boldsymbol{f}$ is called of type (E) (resp. type $(F)$ ) if $N_{p}{ }^{\vee}$ has Property $(E)$ (resp. Property $(F)$ ) at each $p \in M$.

The type of a non-degenerate isometric imbedding defined above just corresponds to the type of the differential operator $L$ associated with $\boldsymbol{f} ; \boldsymbol{f}$ is of type $(E)$ (resp. type $(F)$ ) if and only if $L$ is of elliptic type (resp. finite type) (see $[\mathbf{T n}],[\mathbf{K T}])$. Therefore, as for the space of infinitesimal isometric deformations of $\boldsymbol{f}$, we obtain the following fundamental proposition.

Proposition 1. 2 (see [Tn], [KT]). Let $\boldsymbol{f}$ be a non-degenerate isometric imbedding of a Riemannian manifold $(M, g)$ into a euclidean space $\boldsymbol{R}^{m}$. Then:

(1) If $\boldsymbol{f}$ is of type $(E)$ and $M$ is compact, then the space of global infinitesimal isometric deformations of $\boldsymbol{f}$ is finite dimensional. 
(2) If $\boldsymbol{f}$ is of type $(F)$, then the space of (local) infinitesimal isometric deformations of $\boldsymbol{f}$ is finite dimensional.

\section{§ 2. Canonical isometric imbeddings of symmetric $\boldsymbol{R}$-spaces.}

2. 1. Symmetric $\boldsymbol{R}$-spaces. First we recall the definition of a simple graded Lie algebra of the first kind (=FSGLA).

Definition. Let $\boldsymbol{L}=\sum_{i} \boldsymbol{L}_{i}(i \in \boldsymbol{Z})$ be a finite dimensional graded Lie algebra over $\boldsymbol{R} . \quad \boldsymbol{L}=\sum_{i} \boldsymbol{L}_{i}$ is called an FSGLA if it satisfies

(1) $\boldsymbol{L}$ is non-compact and simple;

(2) $\quad \boldsymbol{L}_{i}=0$ for $|i| \geqq 2$;

(3) $\boldsymbol{L}_{1} \neq 0$.

Let $\boldsymbol{L}=\sum_{i} \boldsymbol{L}_{i}$ be an FSGLA. Then there is a unique element $E$ of $\boldsymbol{L}_{0}$ satisfying $[E, X]=i X$ for $X \in \boldsymbol{L}_{i}(i=-1,0$ and 1$)$. The element $E$ is called the characteristic element of $\boldsymbol{L}=\sum_{i} \boldsymbol{L}_{i}$. As is easily seen, the gradation of $\boldsymbol{L}=\sum_{i} \boldsymbol{L}_{i}$ can be reproduced by the characteristic element $E$. In the following discussion, we represent an FSGLA by the pair $(\boldsymbol{L}, E)$.

Let $(\boldsymbol{L}, E)$ be an FSGLA and $B$ the Killing form of $\boldsymbol{L}$. An involutive automorphism $\theta$ of $\boldsymbol{L}$ is called a standard involution of $(\boldsymbol{L}, E)$ if it satisfies :

(1) $\theta E=-E$;

(2) $\theta$ is a Cartan involution of $\boldsymbol{L}$, i. e., the bilinear form $<$, $>$ of $\boldsymbol{L}$ defined by $\langle X, Y\rangle=-B(X, \theta Y), X, Y \in \boldsymbol{L}$ is positive definite.

As shown in $[\mathbf{K N}]$, there exists a standard involution $\theta$ for each FSGLA $(\boldsymbol{L}, E)$.

LEMMA 2.1 (see $[\mathbf{K N}])$. Let $\theta$ be an standard involution of $(\boldsymbol{L}, E)$. Then :

(1) $\theta \boldsymbol{L}_{i}=\boldsymbol{L}_{-i}(i=-1,0$ and 1$)$.

(2) $\boldsymbol{L}=\boldsymbol{L}_{-1}+\boldsymbol{L}_{0}+\boldsymbol{L}_{1}$ is an orthogonal direct sum with respect to $<,>$.

Let $\theta$ be a standard involution of $(\boldsymbol{L}, E)$. We set

$$
\boldsymbol{G}=\{X \in \boldsymbol{L} ; \theta X=X\}, \boldsymbol{P}=\{X \in \boldsymbol{L} ; \theta X=-X\} .
$$

Then we have

$$
E \in \boldsymbol{P} ;[\boldsymbol{G} \boldsymbol{G}] \subset \boldsymbol{G},[\boldsymbol{G}, \boldsymbol{P}] \subset \boldsymbol{P},[\boldsymbol{P}, \boldsymbol{P}] \subset \boldsymbol{G} .
$$


As is well-known, $\boldsymbol{G}$ is a maximal compact subalgebra of $\boldsymbol{L}$ and $\boldsymbol{L}=\boldsymbol{G}$ $+\boldsymbol{P}$ gives a Cartan decomposition of $\boldsymbol{L}$.

Let $L$ be the adjoint group of $\boldsymbol{L}$. By the map $X \longmapsto \operatorname{ad}(X)(X \in \boldsymbol{L})$ we can regard $L$ as the Lie algebra of $L$. We denote by $G$ the Lie subgroup of $L$ generated by $\boldsymbol{G}$. Then $G$ is a maximal compact subgroup of $L$ and the bilinear form $<,>$ is invariant under the action of $G$, i. e.,

$$
<\operatorname{Ad}(g) X, \operatorname{Ad}(g) Y>=<X, Y>\text { for } X, Y \in \boldsymbol{L}, g \in \boldsymbol{G} \text {. }
$$

We now regard $\boldsymbol{P}$ as a euclidean space with the inner product $<,>\mid \boldsymbol{P}$. Let $M$ be the $\operatorname{Ad}(G)$-orbit in $\boldsymbol{P}$ passing through the characteristic element $E$. Since $\boldsymbol{P}$ is invariant under the action of $G, M$ becomes a compact Riemannian submanifold of $\boldsymbol{P}$.

Let $K$ be the closed subgroup of $G$ defined by

$$
K=\{g \in G ; \operatorname{Ad}(g) E=E\} .
$$

Then $M$ can be represented by a homogeneous space $G / K$. Put $\boldsymbol{K}=\boldsymbol{G} \cap$ $\boldsymbol{L}_{0}$ and $\boldsymbol{M}=\boldsymbol{G} \cap\left(\boldsymbol{L}_{-1}+\boldsymbol{L}_{1}\right)$. Then $\boldsymbol{K}$ can be identified with the Lie algebra of $K$ and $\boldsymbol{M}$ can be identified with the tangent space $T_{0}(G / K)$ at the origin $o(=E)$. By the definition, it is easily observed that:

(1) $[\boldsymbol{K}, \boldsymbol{K}] \subset \boldsymbol{K},[\boldsymbol{K}, \boldsymbol{M}] \subset \boldsymbol{M},[\boldsymbol{M}, \boldsymbol{M}] \subset \boldsymbol{K}$.

(2) $\operatorname{Ad}(k) \boldsymbol{K}=\boldsymbol{K}, \operatorname{Ad}(k) \boldsymbol{M}=\boldsymbol{M}$ for $k \in K$.

(3) $\boldsymbol{K}=\left\{X+\theta X ; X \in \boldsymbol{L}_{0}\right\}, \boldsymbol{M}=\left\{X+\theta X ; X \in \boldsymbol{L}_{-1}\right\}$.

We have

Proposition 2.2 (see [KN], [Tk], [KT]). The homogeneous space G/ $K$ endowed with the induced metric has the structure of a Riemannian symmetric space.

DEFInITION. The Riemannian symmetric space $G / K$ defined above is called the symmetric $R$-space associated with $(\boldsymbol{L}, E)$. The inclusion map $\boldsymbol{f}$ of $G / K$ into $\boldsymbol{P}$ is called the canonical isometric imbedding of $G / K$.

The following proposition is fundamental for the canonical isometric imbeddings of symmetric $R$-spaces.

PROPOSITION 2.3 (see [Tn], [KT]). Let $\boldsymbol{f}$ be the canonical isometric imbedding of a symmetric $R$-space $G / K$. Then:

(1) $\boldsymbol{f}$ is non-degenerate.

(2) Set $\boldsymbol{N}=\boldsymbol{P} \cap \boldsymbol{L}_{0}$. Then $\boldsymbol{N}$ can be identified with the normal vector space $N_{0}$ of $\boldsymbol{f}$ at $0 . \quad \boldsymbol{N}$ is invariant under the action of $\operatorname{Ad}(K)$, i. e., $\operatorname{Ad}(k) \boldsymbol{N}=\boldsymbol{N}$ for $k \in K$. 
(3) For each $A \in \boldsymbol{N}$ the second fundamental form $A^{\vee}$ is given by $\left.A^{\vee}(X, Y)=\langle A,[Y, E]]\right\rangle$ for $X, Y \in M$.

2.2. Satake diagrams. Let $(L, E)$ be an FSGLA and $\theta$ a standard involution of $(\boldsymbol{L}, E)$. In the following discussion, we assume that the complexified Lie algebra $\boldsymbol{L}^{c}$ is simple over $\boldsymbol{C}$. (Under this assumption, the symmetric $R$-space $G / K$ associated with $(L, E)$ is not isomorphic with a Hermitian symmetric space of compact type (see [TK]).)

Let $\boldsymbol{L}=\boldsymbol{G}+\boldsymbol{P}$ be the Cartan decomposition of $\boldsymbol{L}$ obtained by $\theta$ and let $\boldsymbol{a}$ be a maximal abelian subspace of $\boldsymbol{P}$ such that $E \in \boldsymbol{a}$. Let $\boldsymbol{t}$ be a Cartan subalgebra of $\boldsymbol{L}$ containing $\boldsymbol{a}$. Then we have $\boldsymbol{t}=\boldsymbol{a}+\boldsymbol{b}(\boldsymbol{b}=\boldsymbol{t} \cap \boldsymbol{G})$. We set $\boldsymbol{t}_{\boldsymbol{R}}=\boldsymbol{a}+\sqrt{-1} \boldsymbol{b}$. As is easily shown, the Killing form $B$ is positive definite on $\boldsymbol{t}_{\boldsymbol{R}}$. We denote by (, ) the restriction of $B$ to $\boldsymbol{t}_{\boldsymbol{R}}$.

An element $\alpha \in \boldsymbol{t}_{\boldsymbol{R}}$ is called a root if there exists a non-zero vector $Z \in$ $\boldsymbol{L}^{c}$ such that $[H, Z]=(\alpha, H) Z$ for all $H \in \boldsymbol{t}_{\boldsymbol{R}}$. Let $\Delta$ be the set of all nonzero roots. We select and fix a system of vectors $\left\{Z_{\alpha} ; \alpha \in \Delta\right\}$, where $Z_{\alpha}$ is a non-zero root vectors corresponding to $\alpha \in \Delta$.

We now introduce a linear order " $<$ " in $\boldsymbol{t}_{\boldsymbol{R}}$ satisfying: (1) If $(H$, $E)<0$ then $H>0$; (2) If $H \in \boldsymbol{t}_{\boldsymbol{R}} \backslash \sqrt{-1} \boldsymbol{b}$ and $H>0$ then $\theta H<0$. We denote by $\Pi=\left\{\alpha_{1}, \ldots, \alpha_{\ell}\right\} \quad(\ell=$ rank $\boldsymbol{L})$ the set of simple roots with respect to $<$. Under our assumption that $\boldsymbol{L}^{c}$ is simple over $\boldsymbol{C}$, the Dynkin diagram of $\Pi$ is irreducible. Then we have

Proposition 2.4 (see [KN]). (1) For each $\alpha \in \Delta$, it holds $(\alpha, E)=$ $-1,0$ or 1 .

(2) Set $\Delta_{i}=\{\alpha \in \Delta ;(\alpha, E)=i\}(i=-1,0$ or 1$)$. Then:

$$
\begin{aligned}
& \boldsymbol{L}_{i}^{c}=\sum_{\alpha} C Z_{\alpha}\left(\alpha \in \Delta_{i}\right)(i=-1 \text { or } 1) ; \\
& \boldsymbol{L}_{0}^{c}=\boldsymbol{t}^{c}+\sum_{\alpha} C Z_{\alpha}\left(\alpha \in \Delta_{0}\right) .
\end{aligned}
$$

By Proposition 2.4, we easily have

LEMMA 2.5. (1) $\boldsymbol{K}^{c}=\boldsymbol{t}^{c}+\sum_{\alpha} \boldsymbol{C}\left(Z_{\alpha}+\theta Z_{\alpha}\right)\left(\alpha \in \Delta_{0}\right)$.

(2) $\boldsymbol{M}^{c}=\Sigma_{\alpha} \boldsymbol{C}\left(Z_{\alpha}+\theta Z_{\alpha}\right)\left(\alpha \in \Delta_{-1}\right)$

By (1) of Proposition 2.5, we know that there is a unique simple root $\alpha_{i 0} \in \Pi$ such that $\left(\alpha_{i 0}, E\right)=-1 ;\left(\alpha_{i}, E\right)=0$ for $\alpha_{i} \in \Pi \backslash\left\{\alpha_{i 0}\right\}$. The simple root $\alpha_{i 0}$ is called the distinguished root for $(\boldsymbol{L}, E)$. In the Satake diagram for the Riemannian symmetric pair $(L, G)$, the distinguished root can be characterized by :

(a) $\quad \alpha_{i 0}$ is denoted by a white circle;

(b) $\alpha_{i 0}$ is not connected by an arrow with another white circle; 
(c) Let $\mu=\sum_{i} a_{i} \alpha_{i}$ be the highest root of $\Delta$. Then the coefficient of $\alpha_{i 0}$ is equal to 1 , i. e., $a_{i 0}=1$.

In Table I (see Appendix 1), we exhibit all FSGLAs $(\boldsymbol{L}, E)$ with $\boldsymbol{L}^{c}$ simple over $\boldsymbol{C}$ and the symmetric $R$-spaces $G / K$ associated with $(\boldsymbol{L}, E)$. Each FSGLA is represented by the pair of the Satake diagram of $(L, G)$ and the distinguished root; the location of the distinguished root is denoted by a double circle. For the symmetric $R$-spaces, we use the following notation:

$$
\begin{aligned}
& S^{n}=S O(n+1) / S O(n) \text { (the sphere), } \\
& Q^{p, q}(\boldsymbol{R})=\left(S^{p} \times S^{q}\right) / \boldsymbol{Z}_{2}(p, q \geqq 1) \text { (the real quadric), } \\
& G^{p, q}(\boldsymbol{R})=S O(p+q) / S(O(p) \times O(q)) \text { (the real Grassmann manifold), } \\
& G^{p, q}(\boldsymbol{H})=S p(p+q) / S p(p) \times S p(q) \text { (the quaternion Grassmann manifold), } \\
& P^{2}(\boldsymbol{C a y})=F_{4} / S p i n(9) \text { (the Cayley projective plane). }
\end{aligned}
$$

As is known, we have

$$
\begin{aligned}
& G^{1, q}(\boldsymbol{R})=G^{q, 1}(\boldsymbol{R})=P^{q}(\boldsymbol{R}) ; G^{1, q}(\boldsymbol{H})=G^{q, 1}(\boldsymbol{H})=P^{q}(\boldsymbol{H}): \\
& Q^{1,3}(\boldsymbol{R})=Q^{3,1}(\boldsymbol{R})=U(2) ; Q^{2,2}(\boldsymbol{R})=G^{2,2}(\boldsymbol{R}) ; \\
& G^{1,1}(\boldsymbol{H})=P^{1}(\boldsymbol{H})=S^{4} .
\end{aligned}
$$

Here $P^{q}(\boldsymbol{R})$ (resp. $P^{q}(\boldsymbol{H})$ ) means the real (resp. quaternion) projective space.

We now state the main theorem of this papar. The proof will be given in $\S 4$ and $\S 5$.

THEOREM 2.6. Let $(\boldsymbol{L}, E)$ be an FSGLA such that $\boldsymbol{L}^{c}$ is simple over C. Let $G / K$ be the symmetric $R$-space associated with $(L, E)$ and $\boldsymbol{f}$ the canonical isometric imbedding of $G / K$. Then:

(1) $\boldsymbol{f}$ is of type $(F)$ if $G / K \neq Q^{p, q}(\boldsymbol{R})(p, q \geqq 1), P^{n}(\boldsymbol{R})(n \geqq 1)$ nor $G^{2, q}(\boldsymbol{R})(q \geqq 2)$.

(2) $\boldsymbol{f}$ is of type $(E)$ but not of type $(F)$ if $G / K=G^{2, q}(\boldsymbol{R})(q \geqq 3)$.

(3) $\boldsymbol{f}$ is not of type (E) if $G / K=Q^{p, q}(\boldsymbol{R})(p, q \geqq 1)$, or $P^{n}(\boldsymbol{R})(n \geqq$ 1).

Combining Theorem 2. 6 with Proposition 1.2, we have holds :

THEOREM 2.7. Under the same assumptions in Theorem 2.6, it

(1) If $G / K \neq Q^{p, q}(\boldsymbol{R}), P^{n}(\boldsymbol{R})$ nor $G^{2, q}(\boldsymbol{R})(q \geqq 2)$, the space of local infinitesimal isometric deformations of $\boldsymbol{f}$ is finite dimensional.

(2) If $G / K=G^{2, q}(\boldsymbol{R})(q \geqq 3)$, the space of global infinitesimal isometric deformations of $\boldsymbol{f}$ is finite dimensional. 
REMARK. 1). The assertions (1) and (2) in Theorem 2.6 are already obtained in [KT]. There, the determination of type of $\boldsymbol{f}$ is carried out by case-by-case examinations with the help of matrices. In this paper, we will determine the type of $\boldsymbol{f}$ for all symmetric $R$-spaces in a unified manner.

2). If $G / K=P^{n}(\boldsymbol{R})$ the space of global or local infinitesimal isometric deformations of $\boldsymbol{f}$ is infinite dimensional. For the real Grassmann manifolds $G^{2, q}(\boldsymbol{R})(q \geqq 3)$, the dimensions of the spaces of global infinitesimal isometric deformations of $\boldsymbol{f}$ were calculated and the actual global rigidity theorems were established (see $[\mathbf{K T}]$ ).

For some other symmetric $R$-spaces, such as compact Hermitian symmetric spaces and classical compact Lie groups $U(n)(n \geqq 3), S O(n)(n \geqq$ 5) and $S p(n)(n \geqq 1)$, the space of global or local infinitesimal isometric deformations of $\boldsymbol{f}$ were calculated (see the results in [Tn], [Ka]).

\section{$\S 3$. Second fundamental forms of canonical isometric imbeddings.}

Let $G / K$ be a symmetric $R$-space associated with an FSGLA $(\boldsymbol{L}, E)$ such that $\boldsymbol{L}^{c}$ is simple over $\boldsymbol{C}$. Let $\boldsymbol{f}$ be the canonical isometric imbedding of $G / K$. Let $N^{\vee}=N^{\vee}$ o be the space of second fundamental forms of $\boldsymbol{f}$ at the origin $o \in G / K$. Since $\boldsymbol{f}$ is $G$-equivariant, the type of $\boldsymbol{f}$ is determined by the property of $N^{\vee}$.

Before proceeding the study of the property of $\boldsymbol{N}^{\vee}$, we prepare a proposition concerning symmetric bilinear forms.

3. 1. c-decomposable elements. Let $V$ be an $n$-dimensional vector space over $\boldsymbol{R}$. As in 1.2, we denote by $V^{*}$ the dual vector space of $V$ and by $S^{2} V^{*}$ the symmetric product of $V^{*}$.

Let $A \in S^{2} V^{*} . \quad A$ is called $c^{-}$decomposable if it can be expressed as a sum of two decomposable elements in $S^{2} V^{*}$, i. e., $A=\xi_{1} \bullet \xi_{2}+\xi_{3} \bullet \xi_{4}, \xi_{i} \in V^{*}$ $(1 \leqq i \leqq 4)$.

We first prove

Lemma 3.1. Let $W$ be a subspace of $S^{2} V^{*}$. Assume that $W$ contains no non-trivial c-decomposable elements of $S^{2} V^{*}$. Then $W$ has Property $(F)$.

PROOF. Suppose that there are non-zero elements $\xi, \eta \in V^{* c}$ such that $\xi \cdot \eta \in W^{c}$. Write $\xi=\xi_{1}+\sqrt{-1} \xi_{2}, \eta=\eta_{1}+\sqrt{-1} \eta_{2}$, where $\xi_{i}, \eta_{i} \in V^{*}$ $(i=1,2)$. Then the real and imaginary parts of $\xi \cdot \eta$ are given by

$$
\operatorname{Re}(\xi \cdot \eta)=\xi_{1} \cdot \eta_{1}-\xi_{2} \cdot \eta_{2} \in W
$$




$$
\operatorname{Im}(\xi \cdot \eta)=\xi_{1} \cdot \eta_{2}+\xi_{2} \cdot \eta_{1} \in W .
$$

Consequently, $\operatorname{Re}(\xi \cdot \eta)$ and $\operatorname{Im}(\xi \cdot \eta)$ are c-decomposable elements contained in $W$. Since $\xi \cdot \eta \neq 0$, we have $\operatorname{Re}(\xi \cdot \eta) \neq 0$ or $\operatorname{Im}(\xi \cdot \eta) \neq 0$. This implies that $W$ contains a non-trivial c-decomposable element. Thus we obtain the lemma.

Q.E.D.

In the following discussion, it is convenient to characterize $\left(\mathrm{c}^{-}\right)$ decomposable elements by their eigenvalues.

Let $A$ be an element of $S^{2} V^{*}$. Define a symmetric endomorphism $A^{\wedge}$ of $V$ by $A(x, y)=\left\langle x, A^{\wedge}(y)\right\rangle$ for $x, y \in V$, where $\langle$,$\rangle is an inner product$ of $V$. We denote by $r^{+}(A)$ (resp. $r^{-}(A)$ ) the number of positive (resp. negative) eigenvalues of $A^{\wedge}$. Let $\left\{e_{i}\right\}_{1 \leqq i \leqq n}$ be a basis of $V$. By Sylvester's law of inertia, $r^{+}(A)$ (resp. $r^{-}(A)$ ) is equal to the number of positive (resp. negative) eigenvalues of the symmetric matrix $\left(A\left(e_{i}\right.\right.$, $\left.\left.e_{j}\right)\right)_{1 \leq i, j \leq n}$.

Lemma 3.2. Let $A \in S^{2} V^{*}$. Then:

(1) $A$ is decomposable if and only if $r^{ \pm}(A) \leqq 1$.

(2) $A$ is c-decomposable if and only if $r^{ \pm}(A) \leqq 2$.

PROOF. We show the assertion (2). (The assertion (1) is proved in [Tn].) We first assume that $r^{ \pm}(A) \leqq 2$. Then there are non-negative real numbers $a_{i}(1 \leqq i \leqq 4)$ and elements $\xi_{i} \in V^{*}(1 \leqq i \leqq 4)$ such that $A$ can be written in the form

$$
A=a_{1} \xi_{1} \cdot \xi_{1}+a_{2} \xi_{2} \cdot \xi_{2}-a_{3} \xi_{3} \cdot \xi_{3}-a_{4} \xi_{4} \cdot \xi_{4} .
$$

Then we have

$$
\begin{aligned}
A= & \left(\sqrt{a_{1}} \xi_{1}+\sqrt{a_{3}} \xi_{3}\right) \cdot\left(\sqrt{a_{1}} \xi_{1}-\sqrt{a_{3}} \xi_{3}\right) \\
& +\left(\sqrt{a_{2}} \xi_{2}+\sqrt{a_{4}} \xi_{4}\right) \cdot\left(\sqrt{a_{2}} \xi_{2}-\sqrt{a_{4}} \xi_{4}\right) .
\end{aligned}
$$

This implies that $A$ is c-decomposable.

We next show the converse. Let $A=\xi_{1} \cdot \xi_{2}+\xi_{3} \cdot \xi_{4}$ be a c-decomposable element of $S^{2} V^{*}$. Then clearly we have $\operatorname{rank} A^{\wedge} \leqq 4$. Note that there is nothing to prove if $\operatorname{rank} A^{\wedge} \leqq 2$. Now assume the case $\operatorname{rank} A^{\wedge}=4$. Then the elements $\xi_{1}, \xi_{2}, \xi_{3}$ and $\xi_{4}$ are linearly independent. Take a basis $\left\{e_{i}\right\}_{1 \leq i \leq n}$ of $V$ satisfying $\xi_{j}\left(e_{i}\right)=\delta_{j i}, 1 \leqq i \leqq n, 1 \leqq j \leqq 4$. Then it is easily seen that the non-zero eigenvalues of the symmetric matrix $\left(A\left(e_{i}, e_{j}\right)\right)_{1 \leq i, j \leq n}$ are $1,1,-1$ and -1 . Therefore, we have $r^{ \pm}(A)=2$. We next consider the case $\operatorname{rank} A^{\wedge}=3$. Without loss of generality, we may assume that $\xi_{1}, \xi_{2}, \xi_{3}$ are linearly independent and $\xi_{4}$ is written as a linear combination of $\xi_{1}, \xi_{2}, \xi_{3}$, i. e., $\xi_{4}=a \xi_{1}+b \xi_{2}+c \xi_{3}$. Then we have 


$$
A=\left(\xi_{1}+b \xi_{3}\right) \cdot\left(\xi_{2}+a \xi_{3}\right)+(c-a b) \xi_{3} \cdot \xi_{3}
$$

Put $\eta_{1}=\xi_{1}+b \xi_{3}, \eta_{2}=\xi_{2}+a \xi_{3}, \quad \eta_{3}=\xi_{3}$ and take a basis $\left\{f_{i}\right\}_{1 \leqq i \leqq n}$ of $V$ satisfying $\eta_{j}\left(f_{i}\right)=\delta_{j i}, 1 \leqq i \leqq n, 1 \leqq j \leqq 3$. Then it is easy to see that the non-zero eigenvalues of the matrix $\left(A\left(f_{i}, f_{j}\right)\right)_{1 \leq i, j \leq n}$ are $1,-1$ and $c-a b$. This implies $r^{ \pm}(A) \leqq 2$. Thus we obtain the lemma.

Q.E.D.

By Lemmas 3.1 and 3.2, we immediately have

PROPOSITION 3.3. Let $W$ be a subspace of $S^{2} V^{*}$. Then:

(1) $W$ has Property $(E)$ if and only if $W$ contains no non-trivial elements $A$ such that $r^{ \pm}(A) \leqq 1$.

(2) $W$ has Property $(F)$ if $W$ contains no non-trivial elements $A$ such that $r^{ \pm}(A) \leqq 2$.

3. 2 Eigenvalues of second fundamental forms. In the following discussion, we study the property of $\boldsymbol{N}^{\curlyvee}$. For simplicity, we denote by $A^{\wedge}$ the symmetric endomorphism of $\boldsymbol{M}$ associated with $A^{\curlyvee}(A \in \boldsymbol{N})$ and set $r^{+}(A)=r^{+}\left(A^{\vee}\right), r^{-}(A)=r^{-}\left(A^{\smile}\right)$.

We first show

Lemma 3.4. Let $A \in \boldsymbol{N}$. Then $A^{\wedge}=-\operatorname{ad}(E) \cdot \operatorname{ad}(A)$.

Proof. It is easily shown that if $X \in \boldsymbol{P}$ (resp. $Y \in \boldsymbol{G}$ ) then $\operatorname{ad}(X)$ (resp. $\operatorname{ad}(Y))$ is symmetric (resp. skew symmetric) endomorphism of $\boldsymbol{L}$ with respect to $\langle$,$\rangle . Hence by (3) of Proposition 2.3, we have A^{\vee}(X$, $Y)=\langle A,[X,[Y, E]]\rangle=\langle[A, X],[Y, E]\rangle=-\langle[E,[A, X]], Y\rangle$ for $X, Y \in \boldsymbol{M}$. This proves the lemma.

Q.E.D.

LEMMA 3.5. Let $A \in \boldsymbol{N}$. Then $r^{ \pm}(\operatorname{Ad}(k) A)=r^{ \pm}(A)$ for $k \in K$.

Proof. By Lemma 3.4, we easily have $(\operatorname{Ad}(k) A)^{\wedge}=\operatorname{Ad}(k) \cdot A^{\wedge}$. $\operatorname{Ad}(k)^{-1}$ for $k \in K$. Thus our assertion follows immediately. Q. E. D.

Let $\boldsymbol{N}_{1}$ (resp. $\boldsymbol{N}_{2}$ ) be the subset of $\boldsymbol{N}$ consisting of all $A \in \boldsymbol{N}$ such that $A^{\vee}$ is decomposable (resp. c-decomposable). Then by Lemma 3.2, $N_{1}$ and $N_{2}$ can be characterized as follows :

$$
\boldsymbol{N}_{1}=\left\{A \in \boldsymbol{N} ; r^{ \pm}(A) \leqq 1\right\} ; \quad \boldsymbol{N}_{2}=\left\{A \in \boldsymbol{N} ; r^{ \pm}(A) \leqq 2\right\} .
$$

In view of Proposition 3.3, we know that $\boldsymbol{N}^{\vee}$ has Property $(E)$ if and only if $\boldsymbol{N}_{1}=0 ; \boldsymbol{N}^{\vee}$ has Property $(F)$ if $\boldsymbol{N}_{2}=0$.

By the following proposition, we may concentrate our attention on $\boldsymbol{a}$ in order to study the property of $\boldsymbol{N}$. 
Proposition 3.6. Set $\boldsymbol{a}_{1}=\boldsymbol{a} \cap \boldsymbol{N}_{1}, \boldsymbol{a}_{2}=\boldsymbol{a} \cap \boldsymbol{N}_{2}$. Then:

(1) $\boldsymbol{N}^{\vee}$ has Property $(E)$ if and only if $\boldsymbol{a}_{1}=0$.

(2) $\boldsymbol{N}^{\vee}$ has Property $(F)$ if $\boldsymbol{a}_{2}=0$.

ProOF. To show the proposition, if suffices to prove that for each $A$ $\in \boldsymbol{N}$ there exists an element $k \in K$ such that $\operatorname{Ad}(k) A \in \boldsymbol{a}$ (see Lemma 3.5). As shown in $[\mathbf{K N}], \boldsymbol{L}_{0}$ is a reductive Lie algebra whose center is equal to $\boldsymbol{R} E$. Let $\boldsymbol{L}_{0}^{0}$ be the semisimple part of $\boldsymbol{L}_{0}$. Then $\boldsymbol{L}_{0}^{0}=\boldsymbol{K}+\boldsymbol{N}^{0}\left(\boldsymbol{N}^{0}=\boldsymbol{N} \cap\right.$ $\boldsymbol{L}_{0}^{0}$ ) gives a Cartan decomposition of $\boldsymbol{L}_{0}^{0}$ and $\boldsymbol{a}^{0}=\boldsymbol{a} \cap \boldsymbol{L}_{0}^{0}$ is a maximal abelian subspace of $\boldsymbol{N}^{0}$. Let $A \in \boldsymbol{N}$. Then there is an element $B \in \boldsymbol{N}^{0}$ such that $A=B+c E$, where $c \in \boldsymbol{R}$. Since each element of $\boldsymbol{N}^{0}$ is conjugate to an element of $\boldsymbol{a}^{0}$ under the action of $\operatorname{Ad}(K)$, we have $\operatorname{Ad}(k) B \in \boldsymbol{a}^{0}$ for some $k \in K$. Thus, we have $\operatorname{Ad}(k) A \in \boldsymbol{a}$, because $E \in \boldsymbol{a}$ and $\operatorname{Ad}(k) E=E$ for $k \in K$.

Q.E.D.

We now consider the restricted root system of the Riemannian symmetric pair $(L, G)$.

Let $\gamma \in \boldsymbol{a} . \quad \gamma$ is called a restricted root if there is a root $\alpha \in \Delta$ such that $\gamma=\alpha^{-}$, where $\alpha^{-}$denotes the $\boldsymbol{a}$-component of $\alpha$ with respect to the decomposition $\boldsymbol{t}_{\boldsymbol{R}}=\boldsymbol{a}+\sqrt{-1} \boldsymbol{b}$. Let $\Sigma$ be the set of all non-zero restricted roots. For each $\gamma \in \Sigma$, we define an integer $m(\gamma)$ by $m(\gamma)=\#\left\{\alpha \in \Delta ; \alpha^{-}=\right.$ $\gamma\} ; m(\gamma)$ is called the multiplicity of $\gamma$.

We now introduce a linear order in $\boldsymbol{a}$ induced from the linear order "<" in $\boldsymbol{t}_{\boldsymbol{R}}$ by a natural way. Let $\Pi_{\Sigma}=\left\{\gamma_{1}, \ldots, \gamma_{s}\right\} \quad(s=\operatorname{rank}(L / G))$ be the set of simple restricted roots of $\Sigma$ with respect to this linear order. As is easily shown, for each $\gamma_{j} \in \Pi_{\Sigma}$, there is a simple root $\alpha_{i} \in \Pi$ such that $\gamma_{j}=$ $\alpha_{i}^{-}$.

Under the above notation, we have

PROPOSITION 3.7. (see [T/k]). (1) $\Sigma$ is an irreducible reduced root system. Therefore the Dynkin diagram of $\Pi_{\Sigma}$ coincides with one of the Dynkin diagrams of complex simple Lie algebras.

(2) Let $\gamma_{j 0} \in \Pi_{\Sigma}$ satisfy $\gamma_{j 0}=\alpha_{i 0^{-}}$, where $\alpha_{i 0}$ is the distinguished root of $(L, E)$. Then:

(a) $\left(\gamma_{j 0}, E\right)=-1 ; \quad\left(\gamma_{j}, E\right)=0$ for each $\gamma_{j} \in \Pi_{\Sigma}^{0}=\Pi_{\Sigma} \backslash\left\{\gamma_{j 0}\right\}$.

(b) Let $\mu=\Sigma_{i} a_{i} \gamma_{i}$ be the highest root of $\Sigma$. Then $a_{j 0}=1$.

Let us set $\Sigma_{-1}=\left\{\alpha^{-} ; \alpha \in \Delta_{-1}\right\}$. Then, $\Sigma_{-1}$ is given by $\Sigma_{-1}=\{\gamma \in \Sigma ;(\gamma$, $E)=-1\}$.

By use of the multiplicity, $r^{+}(A)$ and $r^{-}(A)$ are calculated as follows : 
Proposition 3.8. Let $A \in \boldsymbol{a}$. Set $R^{+}(A)=\left\{\alpha \in \Sigma_{-1} ;(\alpha, A)>0\right\}$ and $R^{-}(A)=\left\{\alpha \in \Sigma_{-1} ;(\alpha, A)<0\right\}$. Then

$$
\begin{array}{ll}
r^{+}(A)=\Sigma_{\gamma} m(\gamma) & \left(\gamma \in R^{+}(A)\right) ; \\
r^{-}(A)=\Sigma_{\gamma} m(\gamma) & \left(\gamma \in R^{-}(A)\right) .
\end{array}
$$

ProOF. Let $\alpha \in \Delta_{-1}$. Then by Lemma 3.1, we have $A^{\wedge}\left(Z_{\alpha}\right)=(\alpha$, A) $Z_{\alpha}$. On the other hand, since $A^{\wedge} \cdot \theta=\theta \cdot A^{\wedge}$ we have $A^{\wedge}\left(Z_{\alpha}+\theta Z_{\alpha}\right)=(\alpha$, $A)\left(Z_{\alpha}+\theta Z_{\alpha}\right)$. This proves that $(\alpha, A)$ is an eigenvalue of $A^{\wedge}$. Conversely, since $\left\{Z_{\alpha}+\theta Z_{\alpha} ; \alpha \in \Delta_{-1}\right\}$ forms a basis of $\boldsymbol{M}^{c}$ (see Lemma 2.5), the set of eigenvalues of $A^{\wedge}$ is given by $\left\{(\alpha, A) ; \alpha \in \Delta_{-1}\right\}$. It is easy to see that $(\alpha, A)=(\gamma, A)$ holds for each $A \in \boldsymbol{a}$ if $\alpha^{-}=\gamma$. Hence we have the proposition.

Q.E. D.

In Table II (see Appendix 2), we represent the Dynkin diagram of $\Pi_{\Sigma}$ and the multiplicity of $\gamma_{j} \in \Pi_{\Sigma}$ for each $\operatorname{FSGLA}(\boldsymbol{L}, E)$ such that $\boldsymbol{L}^{c}$ is simple over $\boldsymbol{C}$ (cf. [Ar], [H]). There the restricted root $\gamma_{j 0}=\alpha_{i 0}^{-}$is denoted by a double circle.

Let $\gamma \in \Sigma$. Since $\Sigma$ is irreducible, there is at least one simple restricted root $\gamma_{j}$ of the same length, i, e., $(\gamma, \gamma)=\left(\gamma_{j}, \gamma_{j}\right)$. Then, the multiplicity of $\gamma$ is equal to that of $\gamma_{j}$, i. e., $m(\gamma)=m\left(\gamma_{j}\right)$ (see $[\mathbf{H}]$ ).

Making use of Proposition 3.8, we can immediately determine the property of $\boldsymbol{N}^{\vee}$ for those FSGLAs with large multiplicities. In fact, if $m\left(\gamma_{j}\right) \geqq 3$ holds for each $\gamma_{j} \in \Pi_{\Sigma}$ we have $m(\gamma) \geqq 3$. Hence, by Proposition 3.8 we obtain $r^{+}(A) \geqq 3$ or $r^{-}(A) \geqq 3$ for each $A \in \boldsymbol{a} \backslash\{0\}$. This proves $\boldsymbol{a}_{2}=$ 0. Thus, by Proposition 3. 6 we have

PROPOSITION 3.9. Let $(\boldsymbol{L}, E)$ be an FSGLA such that $m\left(\gamma_{j}\right) \geqq 3$ for each $\gamma_{j} \in \Pi_{\Sigma}$. Then $\boldsymbol{N}^{\vee}$ has Property $(F)$.

In $\S 4$, we exhibit all FSGLAs satisfying the assumption of Proposition 3.9 (see Class IV : FSGLAs with large multiplicities).

3. 3. Admissible chains and (c-)decomposable elements. We now consider a method to determine (c-)decomposable elements in $\boldsymbol{N}^{\vee}$ for those FSGLAs with small multiplicities.

Let $\boldsymbol{D}$ be a closed domain in $\boldsymbol{a}$ defined by

$$
\boldsymbol{D}=\left\{A \in \boldsymbol{a} ;\left(\gamma_{j 0}, A\right) \leqq 0 \text { and }\left(\gamma_{j}, A\right) \geqq 0 \text { for } \gamma_{j} \in \Pi_{\Sigma}^{0}\right\} .
$$

Further we put $\boldsymbol{D}_{1}=\boldsymbol{D} \cap \boldsymbol{a}_{1}, \boldsymbol{D}_{2}=\boldsymbol{D} \cap \boldsymbol{a}_{2}$. Then we have

Proposition 3.10. (1) $\boldsymbol{N}^{\vee}$ has Property $(E)$ if and only if $\boldsymbol{D}_{1}=0$.

(2) $\boldsymbol{N}^{\vee}$ has Property $(F)$ if $\boldsymbol{D}_{2}=0$. 
Let $W_{\Sigma}$ be the Weyl group of the restricted root system $\Sigma$. We denote by $W_{\Sigma}^{0}$ the subgroup of $W_{\Sigma}$ generated by the reflections $\left\{\mathrm{S}_{\gamma_{i}} ; r_{i} \in \Pi_{\Sigma}^{0}\right\}$. It is known that for each reflection $S_{\gamma_{i}}\left(\gamma_{i} \in \Pi_{\Sigma}^{0}\right)$ there is an element $k \in K$ such that $S_{\gamma_{i}}=\left.\operatorname{Ad}(k)\right|_{a}$. Hence we have $w(E)=E$ and $r^{ \pm}(w(A))=r^{ \pm}(A)$ for $A$ $\in N, w \in W_{\Sigma}^{0}$ (see Lemma 3.5). Thus, the proposition follows from proposition 3. 6 and the following lemma.

LEMmA 3.11. Let $A \in \boldsymbol{a}$. Then there exists an element $w \in W_{\Sigma}^{0}$ such that $w(A) \in \boldsymbol{D}$ or $-w(A) \in \boldsymbol{D}$.

Proof. We note that $w\left(\Sigma_{-1}\right)=\Sigma_{-1}$ holds for $w \in W_{\Sigma}^{0}$. This can be easily proved by the fact $w(E)=E$ for $w \in W_{\Sigma}^{0}$.

Replacing $A$ by $-A$ if necessary, we may assume that $\left(\gamma_{j 0}, A\right) \leqq 0$. Let $H$ be an element of $\boldsymbol{a}$ such that $\left(\gamma_{j}, H\right)>0$ for each $j \neq j 0$. We difine a function $f$ of $W_{\Sigma}^{0}$ by setting $f(w)=(w(A)-H, w(A)-H)\left(w \in W_{\Sigma}^{0}\right)$. Let $w^{0} \in W_{\Sigma}^{0}$ be an element that gives the minimum of $f$. Then, as is well -known, it holds $\left(\gamma_{j}, w^{0}(A)\right) \geqq 0$ for each $\gamma_{j} \in \Pi_{\Sigma}^{0}$. In order to prove $\left(\gamma_{j 0}\right.$, $\left.w^{0}(A)\right) \leqq 0$, we suppose $\left(\gamma_{j 0}, w^{0}(A)\right)>0$. Since $w^{0}\left(\Sigma_{-1}\right)=\Sigma_{-1}$ and $\gamma_{j 0} \in \Sigma_{-1}$, we have $w^{0}\left(\gamma_{j 0}\right)=\gamma_{j 0}+\Sigma_{j \neq j 0} a_{j} \gamma_{j}$ and $a_{j} \geqq 0 \quad(j \neq j 0)$. Therefore we have

$$
\begin{aligned}
& 0 \geqq\left(\gamma_{j 0}, A\right)=\left(w^{0}\left(\gamma_{j 0}\right), w^{0}(A)\right) \\
& \quad=\left(\gamma_{j 0}, w^{0}(A)\right)+\Sigma_{j \neq j 0} a_{j}\left(\gamma_{j}, w^{0}(A)\right)>0 .
\end{aligned}
$$

This is a contradiction. Hence we have $\left(\gamma_{j 0}, w^{0}(A)\right) \leqq 0$, which proves $w^{0}(A) \in \boldsymbol{D}$.

Q.E.D.

We now introduce a partial order " $<$ " in $\Sigma$ as follows: Let $\alpha=$ $\Sigma_{i} a_{i} \gamma_{i}$ and $\beta=\Sigma_{i} b_{i} \gamma_{i} \in \Sigma$. We write $\alpha<\beta$ when $\alpha \neq \beta$ and $a_{i} \leqq b_{i}$ holds for each $i$. We also define the height $h(\alpha)$ of $\alpha=\Sigma_{i} a_{i} \gamma_{i} \in \Sigma$ by setting $h(\alpha)=$ $\sum_{i} a_{i}$

DEFINITION. An ascending chain $\beta_{1}<\beta_{2}<\cdots<\beta_{r-1}<\beta_{r}$ composed of positive roots of $\Sigma$ is called admissible if the following two conditions are satisfied :

(1) $\beta_{1}=\gamma_{j 0} ; \beta_{r}=\mu(\mu$ is the highest root of $\Sigma)$;

(2) $h\left(\beta_{i+1}\right)=h\left(\beta_{i}\right)+1 \quad(1 \leqq i \leqq r-1)$.

By the definition we have $r=h(\mu)$ and $\beta_{i} \in \Sigma_{-1}$ for $1 \leqq i \leqq r$. The following propositions are useful in the determination of $\boldsymbol{D}_{1}$ and $\boldsymbol{D}_{2}$.

PROPOSITION 3.12. Let $\left\{\beta_{i}(1 \leqq i \leqq r)\right\}$ be an admissible chain in $\Sigma$. Then : 
(1) Assume $r=h(\mu) \geqq 3$ and set $\sigma=\beta_{r-1}-\beta_{2}=\Sigma_{j} a_{j} \gamma_{j}$. Then for each $A \in D_{1}$, it holds $\left(\gamma_{j}, A\right)=0$ if $a_{j} \neq 0$.

(2) Assume $r=h(\mu) \geqq 5$ and set $\varepsilon=\beta_{r-2}-\beta_{3}=\Sigma_{j} b_{j} \gamma_{j}$. Then for each $A \in \boldsymbol{D}_{2}$, it holds $\left(r_{j}, A\right)=0$ if $b_{j} \neq 0$.

Proof. We show (1). Since $A \in \boldsymbol{D}_{1}$, we have $\left(\gamma_{j}, A\right) \geqq 0$ for $j \neq j 0$. Therefore we have $\left(\beta_{1}, A\right) \leqq\left(\beta_{2}, A\right) \leqq \cdots \leqq\left(\beta_{r}, A\right)$. Since $r^{ \pm}(A) \leqq 1$ we have $\left(\beta_{j}, A\right)=0 \quad(2 \leqq j \leqq r-1)$. Consequently, we have $(\sigma, A)=\Sigma_{j} a_{j}\left(\gamma_{j}\right.$, $A)=0$. On the other hand, since $a_{j} \geqq 0(j \neq j 0)$ and $a_{j 0}=0$, we have $\left(\gamma_{j}\right.$, $A)=0$ in case $a_{j} \neq 0$. This completes the proof of (1). The assertion (2) can be similarly dealt with.

Q.E. D.

Proposition 3.13. Let $A \in \boldsymbol{D}$ satisfy $\left(\gamma_{j}, A\right)=0$ for $j(\neq j 0)$. Then:

(1) If $A \in D_{1}$ and $\# \Sigma_{-1} \geqq 2$, then $A=0$.

(2) If $A \in D_{2}$ and $\# \Sigma_{-1} \geqq 3$, then $A=0$.

Proof. Let $\beta \in \Sigma_{-1}$. Then by the assumption, we have $(\beta, A)=\left(\gamma_{j 0}\right.$, $A)$. Therefore if $\left(\gamma_{j 0}, A\right) \neq 0$, then we have $r^{-}(A)=\# \Sigma_{-1}$. Thus our assertion follows from Lemma 3.2.

Q.E. D.

REMARK. As we have seen in the above discussion, admissible chains are useful in the determination of $\boldsymbol{D}_{1}$ and $\boldsymbol{D}_{2}$. However, they are considerably dependent on the restricted root system $\Sigma$ and the location of $\gamma_{j 0}$. Therefore, to select suitable admissible chains for our discussion, we have to consult the list of roots in $[\boldsymbol{B}]$.

In the following example, we consider the cases where the restricted root systems $\Sigma$ are not of classical type. We note that such cases are limited to $\boldsymbol{E} \boldsymbol{I}_{1}$ and $\boldsymbol{E} \boldsymbol{V}_{7}$.

ExAmple. Let $(\boldsymbol{L}, E)$ be $\boldsymbol{E} \boldsymbol{I}_{1}$ or $\boldsymbol{E} \boldsymbol{V}_{7}$. Then, the Dynkin diagram of $\Pi_{\Sigma}^{0}$ is of type $E_{6}$ or $E_{7}$ (see Table II). We note that in this case all the multiplicities are equal to 1 . In view of $[\boldsymbol{B}]$, we have the following:

Case of $\boldsymbol{E I}_{1 .}\left[\begin{array}{l}\mu=\gamma_{1}+2 \gamma_{2}+2 \gamma_{3}+3 \gamma_{4}+2 \gamma_{5}+\gamma_{6} ; \\ h(\mu)=11 ; j 0=1 .\end{array}\right.$
Case of $\boldsymbol{E V}_{7} .\left[\begin{array}{l}\mu=2 \gamma_{1}+2 \gamma_{2}+3 \gamma_{3}+4 \gamma_{4}+3 \gamma_{5}+2 \gamma_{6}+\gamma_{7} ; \\ h(\mu)=17 ; j 0=7 .\end{array}\right.$

Furthermore, for admissible chains of $\Sigma$, we know the following: Let $\left\{\beta_{i}(1 \leqq i \leqq h(\mu))\right\}$ be an admissible chain. Then $\beta_{3}$ and $\beta_{r-2}(r=h(\mu))$ are uniquely determined. They are given as follows : 


$$
\begin{array}{ccc} 
& \beta_{3} & \beta_{r-2} \\
\boldsymbol{E} \boldsymbol{I}_{1} & \gamma_{1}+\gamma_{3}+\gamma_{4} & \mu-\gamma_{2}-\gamma_{4} \\
\boldsymbol{E} \boldsymbol{V}_{7} & \gamma_{5}+\gamma_{6}+\gamma_{7} & \mu-\gamma_{3}-\gamma_{1}
\end{array}
$$

Therefore, the difference $\varepsilon=\beta_{r-2}-\beta_{3}$ is given by $\varepsilon=\gamma_{2}+\gamma_{3}+\gamma_{4}+2 \gamma_{5}+$ $\gamma_{6}$ (Case of $\left.\boldsymbol{E I}_{1}\right), \varepsilon=\gamma_{1}+2 \gamma_{2}+2 \gamma_{3}+4 \gamma_{4}+2 \gamma_{5}+\gamma_{6}$ (Case of $\boldsymbol{E} \boldsymbol{V}_{7}$ ).

Let $A$ be an element of $\boldsymbol{D}_{2}$. Then, by Propositin 3.11 and the above table, we have $\left(\gamma_{j}, A\right)=0$ for $j \neq j 0$. Therefore, by Proposition 3.12 , we obtain $A=0$. This proves $\boldsymbol{D}_{2}=0$. Hence, by Proposition 3.9 we have

Proposition 3.14. Let $(\boldsymbol{L}, E)$ be $\boldsymbol{E I}_{1}$ or $\boldsymbol{E} \boldsymbol{V}_{7}$. Then $\boldsymbol{N}^{\vee}$ has Property $(F)$.

\section{§ 4. Case studies (1).}

As we have seen in the previous section, to determine the types of canonical isometric imbeddings of symmetric $R$-spaces we have only to study the spaces $\boldsymbol{D}_{1}$ and $\boldsymbol{D}_{2}$.

In this and the next sections, we prove the following the theorems.

THEOREM 4.1. Let $(\boldsymbol{L}, E)$ be an FSGLA such that $\boldsymbol{L}$ is simple over $C$ and let $G / K$ be the symmetric $R$-space associated with $(L, E)$. Then:

(1) Assume that $G / K \neq Q^{p, q}(\boldsymbol{R})(p, q \geqq 1), P^{n}(\boldsymbol{R})(n \geqq 1), G^{2, q}(\boldsymbol{R})$ $(q \geqq 3), G^{3,3}(\boldsymbol{R})$ nor $U(3) / O(3)$. Then $\boldsymbol{D}_{2}=0$.

(2) Assume that $G / K=Q^{p, q}(\boldsymbol{R})(p, q \geqq 1)$ or $P^{n}(\boldsymbol{R})(n \geqq 1)$. Then $\boldsymbol{D}_{1} \neq 0$.

For the case $G / K=G^{2, q}(\boldsymbol{R})(q \geqq 3), G^{3,3}(\boldsymbol{R})$ or $U(3) / O(3)$, we have

THEOREM 4.2. Assume that $G / K=G^{2, q}(\boldsymbol{R})(q \geqq 3), G^{3,3}(\boldsymbol{R})$ or $U(3) /$ $O(3)$. Then $\boldsymbol{D}_{1}=0$ but $\boldsymbol{D}_{2} \neq 0$. Moreover:

a). If $G / K=G^{2, q}(\boldsymbol{R})(q \geqq 3), \boldsymbol{N}^{\vee}$ has Property $(E)$ but not Property $(F)$.

b). If $G / K=G^{3,3}(\boldsymbol{R})$ or $U(3) / O(3), \boldsymbol{N}^{\vee}$ has Property $(F)$.

It is easy to see that Theorem 2.6 immediately follows from the above two theorems.

To prove the above thoerems, we divide all the FSGLAs $(\boldsymbol{L}, E)$ into the following five classes.

I . FSGLAs accompanied with real Grassmann manifolds.

II. FSGLAs accompanied with real quadrics.

III. FSGLAs with trivial multiplicities, i. e., all the multiplicities of restricted roots are equal to 1.

IV. FSGLAs with large multiplicities, i.e., all the multiplicities of 
restricted roots are greater than or equal to 3 .

V. Otherwise.

4. 1. Case of Class IV. In view of Table II, we know that Class IV is composed of the following FSGLAs:

$$
\begin{aligned}
& \boldsymbol{A I I}(n)_{p}(1 \leqq p \leqq[n / 2], n \geqq 2), \boldsymbol{B I I}(n)_{1}(n \geqq 2), \boldsymbol{C I I}(n, n)_{n}(n \geqq 2), \\
& \boldsymbol{D I I}(n)_{1}(n \geqq 4), \boldsymbol{E I} \boldsymbol{V}_{1} .
\end{aligned}
$$

The corresponding symmetric $R$-spaces are given as follows :

$$
\begin{aligned}
& G^{p, q}(\boldsymbol{H})(n=p+q, 1 \leqq p \leqq q \leqq[n / 2]), S^{2 n-1}(n \geqq 2), \quad S p(n)(n \geqq 2), \\
& S^{2 n-2}(n \geqq 4), P^{2}(\boldsymbol{C a y}) .
\end{aligned}
$$

As we have seen is 3.2 (Proposition 3.9), for each FSGLA listed above the space $\boldsymbol{N}^{\vee}$ has Property $(F)$. This proves Theorem 4.1 for this class.

4.2 Case of Class I. In view of Table I, we know that Class I is composed of $\boldsymbol{A I}(n)_{p}(n \geqq 1,1 \leqq p \leqq[(n+1) / 2])$. We note that for $\boldsymbol{A I}(n)_{p}$ all the multiplicities are equal to one.

We show

Proposition 4.3. (cf. [KT]). Assume that $(\boldsymbol{L}, E)$ is $\boldsymbol{A I}(n)_{p}(n \geqq 1$, $1 \leqq p \leqq[(n+1) / 2]$ and $(n, p) \neq(5,3))$. Then :

(1) $\boldsymbol{D}_{1} \neq 0$ if $p=1$ or $(n, p)=(3,2)$.

(2) $\boldsymbol{D}_{1}=0$ and $\boldsymbol{D}_{2} \neq 0$ if $p=2$ and $n \geqq 4$.

(3) $\quad \boldsymbol{D}_{2}=0$ if $p \geqq 3$ and $n \geqq 6$.

The case $\boldsymbol{A I}(5)_{3}$ is treated in the next section .

PROOF. We first note that for $\boldsymbol{A I}(n)_{p}$ it holds :

$$
\left[\begin{array}{l}
\mu=\gamma_{1}+\ldots+\gamma_{n} ; \quad h(\mu)=n ; j 0=p . \\
\Sigma_{-1}=\left\{\Sigma_{k=i}^{j} \gamma_{k}(1 \leqq i \leqq p, p \leqq j \leqq n)\right\}
\end{array}\right.
$$

According as the pair $(n, p)(n \geqq 1,1 \leqq p \leqq[(n+1) / 2],(n, p) \neq(5,3))$, we divide this class into the following three cases:

(I-a) The case where $p=1$ and $n \geqq 1$.

(I-b) The case where $p=2$ and $n \geqq 3$.

(I-c) The case where $p \geqq 3$ and $n \geqq 6$.

Case of (I-a). Define an element $A \in \boldsymbol{D}$ by $\left(\gamma_{1}, A\right)=-1,\left(\gamma_{2}, A\right)=1$ and $\left(\gamma_{i}, A\right)=0$ for $i \geqq 3$. In view of the set $\Sigma_{-1}$ we have $r^{+}(A)=0$ and $r^{-}(A)=1$. This clearly implies $A \in \boldsymbol{D}_{1}$. Therefore, we have $\boldsymbol{D}_{1} \neq 0$. 
Case of (I-b). First we assume $n \geqq 4$ and prove $\boldsymbol{D}_{1}=0$ and $\boldsymbol{D}_{2} \neq 0$. In view of the set $\Sigma_{-1}$, we can show that there are three admissible chains $\left\{\beta_{i}\right\},\left\{\beta_{i}^{\prime}\right\}$ and $\left\{\beta_{i}^{\prime \prime}\right\}$ satisfying

$$
\begin{aligned}
& \beta_{2}=\gamma_{1}+\gamma_{2}, \beta_{n-1}=\mu-\gamma_{n} ; \beta_{2}^{\prime}=\gamma_{2}+\gamma_{3}, \\
& \beta_{n-1}^{\prime}=\mu-\gamma_{n} ; \beta_{2}^{\prime \prime}=\gamma_{2}+\gamma_{3}, \beta_{n-1}^{\prime \prime}=\mu-\gamma_{1} .
\end{aligned}
$$

Put $\sigma=\beta_{n-1}-\beta_{2}, \sigma^{\prime}=\beta_{n-1}^{\prime}-\beta_{2}^{\prime}$ and $\sigma^{\prime \prime}=\beta_{n-1}^{\prime \prime}-\beta_{2}^{\prime \prime}$. Then we have $\sigma=\sum_{i=3}^{n-1} \gamma_{i}$ and $\sigma^{\prime}=\gamma_{1}+\sum_{i=4}^{n-1} \gamma_{i}$ and $\sigma^{\prime \prime}=\sum_{i=4}^{n} \gamma_{i}$. Thus, if $A \in D_{1}$ we can easily verify that $\left(\gamma_{i}, A\right)=0$ for $i \neq 2$ (see Proposition 3.12 (1)). Hence by Proposition 3.13 (1) we have $A=0$. This proves $D_{1}=0$.

To show $\boldsymbol{D}_{2} \neq 0$, we consider the element $A \in \boldsymbol{D}$ defined by $\left(\gamma_{2}, A\right)=$ $-1,\left(\gamma_{3}, A\right)=1$ and $\left(\gamma_{i}, A\right)=0(i \neq 2,3)$. Then we can easily see that the non-zero eigenvalues of $A^{\wedge}$ are -1 and -1 . Therefore, we have $r^{-}(A)=$ 2 and $r^{+}(A)=0$, i. e., $A \in \boldsymbol{D}_{2}$. This proves $\boldsymbol{D}_{2} \neq 0$. Now we prove that $\boldsymbol{N}^{\vee}$ has not Property $(F)$. For the element $A$ defined above, there are two suitable covectors $\xi, \eta \in M^{*}$ such that $A^{\vee}=-(\xi \cdot \xi+\eta \cdot \eta)$. Since $\xi$. $\xi+\eta \cdot \eta=(\xi+\sqrt{-1} \eta) \cdot(\xi-\sqrt{-1} \eta), A^{\vee}$ can be regarded as a decomposable element of $S^{2} \boldsymbol{M}^{* c}$. This proves Theorem 4.2 a).

Finally we assume $n=3$. We define an element $A \in \boldsymbol{D}$ by $\left(\gamma_{2}, A\right)=-1$, $\left(\gamma_{1}, A\right)=\left(\gamma_{3}, A\right)=1$. Then it is easy to see that $r^{ \pm}(A)=1$. This implies $A$ $\in D_{1}$. Consequently, we have $\boldsymbol{D}_{1} \neq 0$.

Case of (I-c). First assume that $n \geqq 7$. Then in view of the set $\Sigma_{-1}$, we can show that there are three admissible chaisns $\left\{\beta_{i}\right\},\left\{\beta_{i}^{\prime}\right\}$ and $\left\{\beta_{i}^{\prime \prime}\right\}$ satisfying

$$
\begin{aligned}
& \beta_{3}=\gamma_{p-2}+\gamma_{p-1}+\gamma_{p}, \beta_{n-2}=\mu-\gamma_{n-1}-\gamma_{n} ; \beta_{3}^{\prime}=\gamma_{p}+\gamma_{p+1}+\gamma_{p+2}, \\
& \beta_{n-2}^{\prime}=\mu-\gamma_{n-1}-\gamma_{n} ; \beta_{3}^{\prime \prime}=\gamma_{p}+\gamma_{p+1}+\gamma_{p+2}, \beta_{n-2}^{\prime \prime}=\mu-\gamma_{1}-\gamma_{2} .
\end{aligned}
$$

We put $\varepsilon=\beta_{n-2}-\beta_{3}, \varepsilon^{\prime}=\beta_{n-2}^{\prime}-\beta_{3}^{\prime}$ and $\varepsilon^{\prime \prime}=\beta_{n-2}^{\prime \prime}-\beta_{3}^{\prime \prime}$. Then we have $\varepsilon=$ $\sum_{i=1}^{p-3} \gamma_{i}+\sum_{i=p+1}^{n-2} \gamma_{i}, \varepsilon^{\prime}=\sum_{i=1}^{p-1} \gamma_{i}+\sum_{i=p+3}^{n-2} \gamma_{i}$, and $\varepsilon^{\prime \prime}=\sum_{i=3}^{p-1} \gamma_{i}+\sum_{i=p+3}^{n} \gamma_{i}$. Therefore, for each $A \in \boldsymbol{D}_{2}$ we can easily verify that $\left(\gamma_{i}, A\right)=0$ for $i \neq p$ (see Proposition $3.12(2)$ ). Hence by Proposition 3.13 (2), we have $A=0$. This proves $\boldsymbol{D}_{2}=0$.

We next assume $n=6$. Then we have $p=3$ and $\gamma_{i}=\mu-\sum_{j \neq i} \gamma_{j}$ for each $i(1 \leqq i \leqq 6)$. Thus it can be easily shown that if $i \neq 3$ then there is an admissible chain $\left\{\beta_{i}\right\}$ such that $\gamma_{i}=\beta_{n-2}-\beta_{3}$. Therefore we have $\left(\gamma_{i}, A\right)=$ $0(i \neq 3)$ for each $A \in \boldsymbol{D}_{2}$. Thus, as in the case $n \geqq 7$, we can prove $\boldsymbol{D}_{2}=0$.

By the above discussion, we have proved Proposition 4.3. Q.E.D.

4. 3. Case of Class II. In view of Table II, we know that Class II is composed of $\boldsymbol{B I}(n, p)_{1}(n \geqq 2,2 \leqq p \leqq n), \boldsymbol{D I}(n, p)_{1}(n \geqq 4,2 \leqq p \leqq n)$ and $\boldsymbol{A I I I}(2,2)_{2}$ (Here we note the identity $U(2)=Q^{1,3}(\boldsymbol{R})$ ). 
We show

PROPOSITION 4.4 (cf. [KT]). Assume that $(\boldsymbol{L}, E)$ is $\boldsymbol{B I}(n, p)_{1}$ $(n \geqq 2,2 \leqq p \leqq n), \boldsymbol{D I}(n, p)_{1}(n \geqq 4,2 \leqq p \leqq n)$ or $\boldsymbol{A I I I}(2,2)_{2}$. Then $\boldsymbol{D}_{1} \neq 0$.

Proof. We prove the case where $(\boldsymbol{L}, E)$ is $\boldsymbol{B I}(n, p)_{1}$ or $\boldsymbol{D I}(n, p)_{1}$. The case where $(\boldsymbol{L}, \boldsymbol{E})=\boldsymbol{A I I I}(2,2)_{2}$ is similary dealt with.

In view of $[\boldsymbol{B}]$, we have the following:

The case where $\boldsymbol{B I}(n, p)_{1}(p \leqq n)$ or $\boldsymbol{D I}(n, p)_{1}(p \neq n-1)$ :

$$
\left[\begin{array}{c}
\mu=\gamma_{1}+2\left(\gamma_{2}+\ldots+\gamma_{p}\right) ; h(\mu)=2 p-1 ; j 0=1 . \\
\sum_{-1}=\left\{\sum_{k=1}^{i} \gamma_{k} \quad(1 \leqq i \leqq p) ;\right. \\
\left.\sum_{k=1}^{i} \gamma_{k}+2 \sum_{\ell=i+1}^{p} \gamma_{\ell} \quad(1 \leqq i \leqq p-1)\right\} .
\end{array}\right.
$$

The case where $\boldsymbol{D I}(n, n)_{1}$ :

$$
\left[\begin{array}{c}
\mu=\gamma_{1}+2\left(\gamma_{2}+\ldots+\gamma_{n-2}\right)+\gamma_{n-1}+\gamma_{n} ; h(\mu)=2 n-3 ; j 0=1 \\
\sum_{-1}=\left\{\sum_{k=1}^{i} \gamma_{k} \quad(1 \leqq i \leqq n) ; \sum_{k=1}^{n-2} \gamma_{k}+\gamma_{n} ;\right. \\
\left.\sum_{k=1}^{i} \gamma_{k}+2 \sum_{\ell=i+1}^{n-2} \gamma_{k}+\gamma_{n-1}+\gamma_{n} \quad(1 \leqq i \leqq n-3)\right\} .
\end{array}\right.
$$

Let us define an element $A \in \boldsymbol{D}$ by $\left(\gamma_{1}, A\right)=-1,\left(\gamma_{2}, A\right)=1$ and $\left(\gamma_{i}\right.$, $A)=0$ for $i \geqq 3$. Then we can easily see that $(\gamma, A)=0$ holds for each $\gamma \in \Sigma_{-1}$ such that $\gamma \neq \gamma_{1}, \mu$. Since $\left(\gamma_{1}, \gamma_{1}\right)=(\mu, \mu)$, it follows that $m(\mu)=$ $m\left(\gamma_{1}\right)=1$. Thus, we have $r^{ \pm}(A)=1$, which implies $A \in \boldsymbol{D}_{1}$. This proves $D_{1} \neq 0$.

Q.E. D.

4. 4. Case of Class III. In view of Table II, we know that Class III is composed of $\boldsymbol{A I}(n)_{p}, \boldsymbol{B D} \boldsymbol{I}(n, n)_{1}, \boldsymbol{C I}(n)_{n}(n \geqq 3), \boldsymbol{D I}(n, n)_{n}(n \geqq 5)$ $\boldsymbol{E I}_{1}$ and $\boldsymbol{E} \boldsymbol{V}_{7}$. We note that the case where $(\boldsymbol{L}, E)$ is $\boldsymbol{A I}(n)_{p}, \boldsymbol{B D} \boldsymbol{I}(n, n)_{1}$, $E I_{1}$ or $E V_{7}$ has been already treated in 3.3, 4.2 and 4.3 (see Propositions 3. $14,4.3$ and 4.4).

We now prove

Proposition 4.5. Let $(\boldsymbol{L}, E)$ be one of $\boldsymbol{C I}(n)_{n}(n \geqq 4), \boldsymbol{D I}(n, n)_{n}$ $(n \geqq 5)$. Then $\boldsymbol{D}_{2}=0$.

(The case where $(\boldsymbol{L}, E)$ is $\boldsymbol{C I}(3)_{3}$ is treated in the next section.)

PROOF. (III-a) Case of $\boldsymbol{C I}(n)_{n}(n \geqq 4)$. In view of $[\boldsymbol{B}]$, we have the following :

$$
\left[\begin{array}{l}
\mu=2\left(\gamma_{1}+\ldots+\gamma_{n-1}\right)+\gamma_{n} ; h(\mu)=2 n-1 ; j 0=n . \\
\sum_{-1}=\left\{\sum_{k=i}^{n} \gamma_{k} \quad(1 \leqq i \leqq n) ;\right. \\
\left.\sum_{k=i}^{j} \gamma_{k}+2 \sum_{\ell=j+1}^{n-1} \gamma_{\ell}+\gamma_{n} \quad(1 \leqq i \leqq j \leqq n-2)\right\} .
\end{array}\right.
$$

Since $n \geqq 4$, there are two admissible chains $\left\{\beta_{i}\right\}$ and $\left\{\beta_{i}^{\prime}\right\}$ such that 
$\beta_{3}=\gamma_{n-2}+\gamma_{n-1}+\gamma_{n}, \beta_{2 n-3}=\mu-2 \gamma_{1} ; \beta_{3}^{\prime}=2 \gamma_{n-1}+\gamma_{n}, \beta_{2 n-3}^{\prime}=\mu-\gamma_{1}-\gamma_{2}$. We put $\varepsilon=\beta_{2 n-3}-\beta_{3}, \varepsilon^{\prime}=\beta_{2 n-3}^{\prime}-\beta_{3}^{\prime}$. Let $A \in \boldsymbol{D}_{2}$. Since $\varepsilon=2\left(\gamma_{2}+\ldots+\gamma_{n-3}\right)+\gamma_{n-2}+$ $\gamma_{n-1}$ and $\varepsilon^{\prime}=\gamma_{1}+\gamma_{2}+2\left(\gamma_{3}+\ldots+\gamma_{n-2}\right)$, we have $\left(\gamma_{i}, A\right)=0$ for $i \neq n$ (see Proposition $3.12(2)$ ). Hence by Proposition 3.12 (2) we have $A=0$. This proves $\boldsymbol{D}_{2}=0$.

(III-b) Case of $\boldsymbol{D I}(n, n)_{n}(n \geqq 5)$. We first note the following :

$$
\left[\begin{array}{rl}
\mu= & \gamma_{1}+2\left(\gamma_{2}+\ldots+\gamma_{n-2}\right)+\gamma_{n-1}+\gamma_{n} ; h(\mu)=2 n-3 ; j 0=n . \\
\sum_{-1}= & \left\{\sum_{k=i}^{n} \gamma_{k} \quad(1 \leqq i \leqq n) ;\right. \\
& \sum_{k=i}^{n-2} \gamma_{k}+\gamma_{n}(1 \leqq i \leqq n-2) ; \\
& \left.\sum_{k=i}^{j} \gamma_{k}+2 \sum_{\ell=j+1}^{n-2} \gamma_{\ell}+\gamma_{n-1}+\gamma_{n}(1 \leqq i \leqq j \leqq n-3)\right\} .
\end{array}\right.
$$

Then we can easily show that there are two admissible chains $\left\{\beta_{i}\right\}$ and $\left\{\beta_{i}^{\prime}\right\}$ satisfying $\beta_{3}=\gamma_{n-2}+\gamma_{n-1}+\gamma_{n}, \beta_{2 n-5}=\mu-\gamma_{2}-\gamma_{3} ; \beta_{3}^{\prime}=\gamma_{n-3}+\gamma_{n-2}+\gamma_{n}$, $\beta_{2 n-5}^{\prime}=\mu-\gamma_{1}-\gamma_{2}$. We put $\varepsilon=\beta_{2 n-5}-\beta_{3}$ and $\varepsilon^{\prime}=\beta_{2 n-5}^{\prime}-\beta_{3}^{\prime}$. Then we have $\varepsilon=\gamma_{1}+\gamma_{2}, \varepsilon^{\prime}=\gamma_{3}+\gamma_{4}$ in case $n=5$ and $\varepsilon=\sum_{i=1}^{3} \gamma_{i}+2 \sum_{j=4}^{n-3} \gamma_{j}+\gamma_{n-2}, \quad \varepsilon^{\prime}=\gamma_{2}+$ $2 \sum_{i=3}^{n-4} \gamma_{i}+\gamma_{n-3}+\gamma_{n-2}+\gamma_{n-1}$ in case $n \geqq 6$. Let $A \in \boldsymbol{D}_{2}$. Then by Proposition 3.12 (2) we have $\left(\gamma_{i}, A\right)=0$ for $i \neq n$. Thus we have $A=0$ (see Proposition $3.13(2))$. This implies that $\boldsymbol{D}_{2}=0$.

Thus, we obtain the proposition.

Q.E.D.

4. 4. Case of Class V. In view of Table I and Table II, we know that Class $\mathrm{V}$ is composed of $\boldsymbol{A I I I}(n, n)_{n}(n \geqq 3), \boldsymbol{D I I I}(n)_{n}(n \geqq 3)$ and $\boldsymbol{E} \boldsymbol{V I I}_{3}$. The corresponding symmetric $R$-spaces are given by $U(n)(n \geqq$ 3), $U(2 n) / S p(n)(n \geqq 3)$ and $E_{6} \cdot S O(2) / F_{4}$.

We now prove

Proposition 4.6. Let $(\boldsymbol{L}, E)$ be one of $\boldsymbol{A I I I}(n, n)_{n}(n \geqq 4)$, $\boldsymbol{D I I I}(n)_{n}(n \geqq 4)$. Then $\boldsymbol{D}_{2}=0$.

Proof. We note that in case $\boldsymbol{A I I I}(n, n)_{n}(n \geqq 4)$ and $\boldsymbol{D I I I}(n)_{n}(n \geqq$ 4) the fundamental system $\Pi_{\Sigma}$ is isomorphic with that of $\boldsymbol{C I}(n)_{n}(n \geqq 4)$. Hence, by the discussion in 4.4 (III-a), we have $\boldsymbol{D}_{2}=0$.

Q.E.D.

The following three cases: $\boldsymbol{A I I I}(3,3)_{3} ; \boldsymbol{D I I I}(3)_{3}$ and $\boldsymbol{E} \boldsymbol{V I I} I_{3}$ are treated in the next section.

\section{$\S 5$. Case studies (2).}

In this section, we complete the proofs of Theorems 4.1 and 4.2.

5. 1. Case of $\boldsymbol{A I}(5)_{3}$ or $\boldsymbol{C I}(3)_{3}$. Here we assume that $(\boldsymbol{L}, E)$ is $\boldsymbol{A I}(5)_{3}$ or $\boldsymbol{C I}(3)_{3}$ and show Theorem 4.2. We note that the corresponding 
symmetric $R$-space is $G^{3,3}(\boldsymbol{R})$ or $U(3) / O(3)$.

In view of Table I, the Satake diagram of the symmetric pair $(L, G)$ contains no black circles and no arrows. Thus, we know that $\boldsymbol{t}_{\boldsymbol{R}}=\boldsymbol{a}$ and hence $\Sigma=\Delta, \theta \alpha=-\alpha$ for $\alpha \in \Sigma$. Therefore we can select root vectors $\left\{Z_{\alpha}\right.$; $\alpha \in \Delta\}$ of $\boldsymbol{L}^{c}$ satisfying :

$$
\text { (1) } \left.Z_{\alpha} \in L ; （ 2\right) \quad \theta Z_{\alpha}=Z_{-\alpha} ; \text { (3) }\left\langle Z_{\alpha}, Z_{\alpha}\right\rangle=1 / 2 \text {. }
$$

Now set $X_{\alpha}=Z_{\alpha}+\theta Z_{\alpha}$ for $\alpha \in \Delta_{-1}$. Then we know that $\left\{X_{\alpha} ; \alpha \in \Delta_{-1}\right\}$ forms an orthonormal basis of $\boldsymbol{M}$ with respect to $\langle$,$\rangle .$

We first show

LEMMA 5.1. Let $\alpha, \beta \in \Delta_{-1}$ satisfy $\alpha \pm \beta \notin \Delta \cup\{0\}$. Then it holds

$$
A^{\vee}\left(X_{\alpha}, X_{\beta}\right)=0 \text { for } A \in N \text {. }
$$

Proof. By the definition of $X_{\alpha}$ and $X_{\beta}$, we have

$$
\left[X_{\alpha},\left[X_{\beta}, E\right]\right]=\left[Z_{\alpha}+Z_{-\alpha},-Z_{\beta}+Z_{-\beta}\right]=0 .
$$

Then our assertion follows from Proposition 2. 4.

Q.E.D.

We now show

Lemma 5.2. Asume that $(\boldsymbol{L}, E)$ is $\boldsymbol{A I}(5)_{3}$ or $\boldsymbol{C I}(3)_{3}$. Let $A \in \boldsymbol{a}$. Then $A \in \boldsymbol{D}_{2}$ if and only if there is a non-positive number $a \in \boldsymbol{R}$ such that

(1) In case $\boldsymbol{A I}(5)_{3}$, it holds one of the following:

(i ) $\left(\gamma_{3}, A\right)=a,\left(\gamma_{2}, A\right)=\left(\gamma_{5}, A\right)=-a,\left(\gamma_{1}, A\right)=\left(\gamma_{4}, A\right)=0$;

(ii) $\left(\gamma_{3}, A\right)=a,\left(\gamma_{1}, A\right)=\left(\gamma_{4}, A\right)=-a,\left(\gamma_{2}, A\right)=\left(\gamma_{5}, A\right)=0$,

(2) In case $\boldsymbol{C I}(3)_{3}$, it holds $\left(\gamma_{1}, A\right)=\left(\gamma_{2}, A\right)=-a$ and $\left(\gamma_{3}, A\right)=2 a$.

ProOF. We first consider the case $\boldsymbol{A I}(5)_{3}$. As in the proof of Proposition 4.3 , we can verify that there are three admissible chains $\left\{\beta_{i}\right\},\left\{\beta_{i}^{\prime}\right\}$ and $\left\{\beta_{i}^{\prime \prime}\right\}$ satisfying $\beta_{3}=\gamma_{1}+\gamma_{2}+\gamma_{3} ; \beta_{3}^{\prime}=\gamma_{2}+\gamma_{3}+\gamma_{4} ; \beta_{3}^{\prime \prime}=\gamma_{3}+\gamma_{4}+\gamma_{5}$. Now assme $A \in D_{2}$. Then we have $\left(\beta_{3}, A\right)=\left(\beta_{3}^{\prime}, A\right)=\left(\beta_{3}^{\prime \prime}, A\right)=0$. Consequently, we have $\left(\gamma_{1}+\gamma_{2}+\gamma_{3}, A\right)=0,\left(\gamma_{1}, A\right)=\left(\gamma_{4}, A\right)$ and $\left(\gamma_{2}, A\right)=\left(\gamma_{5}, A\right)$. Put $a=$ $\left(\gamma_{3}, A\right)$. If $a=0$, then we easily obtain $A=0$, because $\left(\gamma_{i}, A\right) \geqq 0$ for $i(\neq 3)$. Next assume $a<0$. Then since $\gamma_{3} \in R^{-}(A)$ and $r^{-}(A) \leqq 2$, we have $\gamma_{2}+\gamma_{3}$ $\notin R^{-}(A)$ or $\gamma_{3}+\gamma_{4} \notin R^{-}(A)$. Assume the case $\gamma_{2}+\gamma_{3} \notin R^{-}(A)$. Then we have $\left(\gamma_{2}+\gamma_{3}, A\right) \geqq 0$. Since $\left(\gamma_{1}+\gamma_{2}+\gamma_{3}, A\right)=0$ and $\left(\gamma_{1}, A\right) \geqq 0$, it follows that $\left(\gamma_{2}+\gamma_{3}, A\right)=0$. Hence we have $\left(\gamma_{1}, A\right)=\left(\gamma_{4}, A\right)=0$ and $\left(\gamma_{2}, A\right)=\left(\gamma_{5}\right.$, $A)=-a$. Similarly, in case $\gamma_{3}+\gamma_{4} \notin R^{-}(A)$, we have $\left(\gamma_{2}, A\right)=\left(\gamma_{5}, A\right)=0$ and $\left(\gamma_{1}, A\right)=\left(\gamma_{4}, A\right)=-a$. This proves (1).

Conversely, assume that there is a negative number $a$ satisfying (i) or 
(ii). Then we can easily observe that $r^{ \pm}(A)=2$. This proves $A \in \boldsymbol{D}_{2}$.

We next consider the case $\boldsymbol{C I}(3)_{3}$. As in the proof of Proposition 4.5 (III-a), we can easily verify that the following two admissible chains are possible :

$$
\begin{aligned}
& \gamma_{3}<\gamma_{2}+\gamma_{3}<\gamma_{1}+\gamma_{2}+\gamma_{3}<\gamma_{1}+2 \gamma_{2}+\gamma_{3}<\mu ; \\
& \gamma_{3}<\gamma_{2}+\gamma_{3}<2 \gamma_{2}+\gamma_{3}<\gamma_{1}+2 \gamma_{2}+\gamma_{3}<\mu .
\end{aligned}
$$

Therefore, if $A \in \boldsymbol{D}_{2}$, we have $\left(\gamma_{1}+\gamma_{2}+\gamma_{3}, A\right)=\left(2 \gamma_{2}+\gamma_{3}, A\right)=0$. Put $a=$ $\left(\gamma_{3}, A\right) / 2$. Then we have $\left(\gamma_{1}, A\right)=\left(\gamma_{2}, A\right)=-a$. This proves (2). The converse can be easily verified. We have thus obtained the lemma. Q.E.D.

As a consequence of the above lemma, we easily have

LEMMA 5.3. Let $(\boldsymbol{L}, E)$ be $\boldsymbol{A I}(5)_{3}$ or $\boldsymbol{C I}(3)_{3}$. Then it holds that $D_{1}=0$ and $D_{2} \neq 0$.

The proof of Lemma 5.3 is left to the reader.

We now give the proof of Theorem 4.2.

Proof of THEOREM 4.2. Here we show the case of $\boldsymbol{C I}(3)_{3}$. (The case where $(\boldsymbol{L}, E)$ is $\boldsymbol{A I}(5)_{3}$ is similarly dealt with; hence we omit the proof for this case.)

We first suppose that $\boldsymbol{N}^{\vee}$ has not Property $(F)$. Let $C^{\vee}$ be a non -trivial decomposable element of $N^{\vee c}$. Write $C=A+\sqrt{-1} B$, where $A$, $B \in N$. As we have seen in the proof of Lemma 3.1, both $A^{\vee}$ and $B^{\vee}$ are c-decomposable elements of $\boldsymbol{N}^{\vee}$. Thus, without loss of generality, we may assume that $A \neq 0$ and $A \in \boldsymbol{D}_{2}$ (see the discussion in $\S 3$ ). We may further assume that $\left(\gamma_{1}, A\right)=\left(\gamma_{2}, A\right)=1$ and $\left(\gamma_{3}, A\right)=-2$ (see Lemma 5. 2). Now set $\sigma_{1}=\gamma_{3}, \sigma_{2}=\gamma_{2}+\gamma_{3}, \sigma_{3}=\mu-\gamma_{1}=\gamma_{1}+2 \gamma_{2}+\gamma_{3}$ and $\sigma_{4}=\mu=2 \gamma_{1}+2 \gamma_{2}+\gamma_{3}$. Then we have $\left(\sigma_{1}, A\right)=-2,\left(\sigma_{2}, A\right)=-1,\left(\sigma_{3}, A\right)=1$ and $\left(\sigma_{4}, A\right)=2$. Put $X_{i}=X_{\sigma_{i}}$ for $i(1 \leqq i \leqq 4)$ and set $\boldsymbol{M}_{1}=\sum_{i=1}^{4} \boldsymbol{R} X_{i}$. It is easily seen that the restriction of $C^{\vee}$ to $\boldsymbol{M}_{1}^{c}$ is also decomposable. Thus, for the complex symmetric matrix $C_{1}$ defined by $C_{1}=\left(C^{\vee}\left(X_{i}, X_{j}\right)\right)_{1 \leq i, j \leq 4}$, it holds $\operatorname{rank}_{C} C_{1} \leqq 2$. Define a real symmetric matrix $A_{1}$ by $A_{1}=\left(A^{\vee}\left(X_{i}, X_{j}\right)\right)_{1 \leq i, j \leq 4}$. Then $A_{1}$ is the real part of the complex matrix $C_{1}$. Moreover, since $A^{\wedge}\left(X_{i}\right)=\left(\sigma_{i}, A\right)$ $X_{i}(1 \leqq i \leqq 4)$, we know that the matrix $A_{1}$ is a diagonal matrix whose diagonal components are given by

$$
\begin{array}{ll}
A^{\curlyvee}\left(X_{1}, X_{1}\right)=-2, & A^{\curlyvee}\left(X_{2}, X_{2}\right)=-1, \\
A^{\smile}\left(X_{3}, X_{3}\right)=1, & A^{\curlyvee}\left(X_{4}, X_{4}\right)=2 .
\end{array}
$$

On the other hand, since $\sigma_{1} \pm \sigma_{3} \notin \Delta \cup\{0\}, \sigma_{1} \pm \sigma_{4} \notin \Delta \cup\{0\}$ and $\sigma_{2} \pm \sigma_{4} \notin \Delta \cup$ $\{0\}$, the matrix $C_{1}$ can be written in the following form: 


$$
C_{\mathrm{l}}=\left(\begin{array}{llll}
a & f & 0 & 0 \\
f & b & g & 0 \\
0 & g & c & h \\
0 & 0 & h & d
\end{array}\right),
$$

where $a \sim h$ are complex numbers satisfying

$$
\begin{aligned}
& \operatorname{Re} a=-2, \operatorname{Re} b=-1, \operatorname{Re} c=1, \operatorname{Re} d=2 ; \\
& \operatorname{Re} f=\operatorname{Re} g=\operatorname{Re} h=0 .
\end{aligned}
$$

Note that the $(2,2)$-cofactor of $C_{1}$ is given by $a\left(c d-h^{2}\right)$. However, by the above equations, we can show that $a\left(c d-h^{2}\right) \neq 0$. This implies that $\operatorname{rank}_{C} C_{1} \geqq 3$, contradicting our assumption.

Q.E. D.

5. 2. Case of $\boldsymbol{A I I I}(3,3)_{3}, \boldsymbol{D I I I}(3)_{3}$ or $\boldsymbol{E V I I}{ }_{3}$. Finally, we prove

Proposition 5.4. Let $(\boldsymbol{L}, E)$ be one of $\boldsymbol{A I I I}(3,3)_{3}, \boldsymbol{D I I I}(3)_{3}$ and $\boldsymbol{E V I I}_{3}$. Then $\boldsymbol{D}_{2}=0$.

PROOF. We note that in this setting, $\Pi_{\Sigma}$ coincides with the fundamental system of restricted roots associated with $\boldsymbol{C I}(3)_{3}$. Let $A \in \boldsymbol{D}_{2}$. Suppose that $A \neq 0$. Then by Lemma 5.2(2), there is a negative number $a \in \boldsymbol{R}$ such that $\left(\gamma_{1}, A\right)=\left(\gamma_{2}, A\right)=-a$ and $\left(\gamma_{3}, A\right)=2 a$. Put $\gamma=\gamma_{2}+\gamma_{3}$. Then we have $\gamma \in \Sigma_{-1}$ and $(\gamma, A)=a$. This proves $\gamma \in R^{-}(A)$. On the other hand, from the Dynkin diagram of $\Pi_{\Sigma}$, we know that $\left(\gamma_{3}, \gamma_{3}\right)=2\left(\gamma_{2}, \gamma_{2}\right)$ and $\left(\gamma_{2}\right.$, $\left.\gamma_{3}\right)=-\left(\gamma_{2}, \gamma_{2}\right)$. Hence we have $(\gamma, \gamma)=\left(\gamma_{2}, \gamma_{2}\right)$ and hence $m(\gamma)=m\left(\gamma_{2}\right)$. In view of Table II, we have $m\left(\gamma_{2}\right)=2$ in case $\boldsymbol{A I I I}(3,3)_{3}, m\left(\gamma_{2}\right)=4$ in case $\boldsymbol{D I I I}(3)_{3}$ and $m\left(\gamma_{2}\right)=8$ in case $\boldsymbol{E V I I I}_{3}$. Then by Proposition 3.8, we have $r^{-}(A) \geqq m(\gamma)+m\left(\gamma_{3}\right) \geqq 3$. This contradicts our assumption $A \in \boldsymbol{D}_{2}$. Therefore we have $\boldsymbol{D}_{2}=0$.

Q.E.D.

\begin{tabular}{|c|c|c|}
\hline FSGLA & Satake Diagram & $R$-space $\quad G / K$ \\
\hline $\begin{array}{l}\boldsymbol{A I}(n)_{p} \\
{\left[\begin{array}{l}n \geqq 1 \\
1 \leqq p \leqq[(n+1) / 2\end{array}\right]}\end{array}$ & 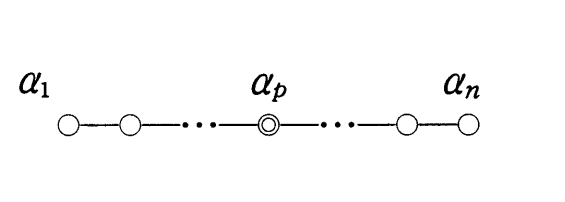 & $G^{p, q}(\boldsymbol{R})(p+q=n+1)$ \\
\hline $\begin{array}{l}\boldsymbol{A I I}(n)_{p} \\
{\left[\begin{array}{l}n \geqq 2 \\
1 \leqq p \leqq[n / 2]\end{array}\right]}\end{array}$ & $. \bullet-\alpha_{2 p} \bullet \ldots \multimap \stackrel{\alpha_{2 n-2}}{\bullet}$ & $G^{p, q}(\boldsymbol{H})(p+q=n)$ \\
\hline
\end{tabular}

Thus, by Propositions 3.14, 4. 3-4. 6 and 5.4, we obtain Theorem 4.1.

Appendix 1. Table I (FSGLAs and symmetric $R$-spaces) 


$\begin{array}{llll}\boldsymbol{A I I I}(n, n)_{n} & \overbrace{(n \geqq 2)}^{\alpha_{1}} & & \\ & \alpha_{n-1} & & \\ \alpha_{2 n-1} & \alpha_{n+1} & \\ \end{array}$

$$
\boldsymbol{B I}(n, p)_{1}
$$

$\left[\begin{array}{l}n \geqq 2 \\ 2 \leqq p \leqq n\end{array}\right]$

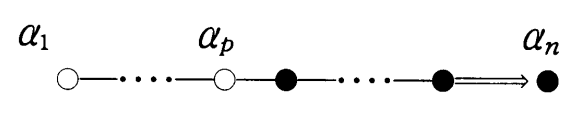

$Q^{p-1, q-1}(\boldsymbol{R})$

$$
(p+q=2 n+1)
$$

\begin{tabular}{l}
$\boldsymbol{B I I}(n)_{1}$ \\
$(n \geqq 2)$ \\
\hline $\boldsymbol{C I}(n)_{n}$ \\
$(n \geqq 3)$ \\
$\boldsymbol{C I I}(n, n)_{n}$ \\
$(n \geqq 2)$
\end{tabular}

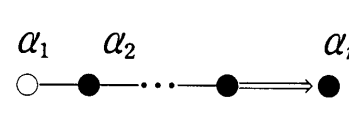

$S^{2 n-1}$

\section{$\alpha_{1} \_\circ \ldots \multimap \rightleftharpoons \alpha_{n}$}

$U(n) / O(n)$

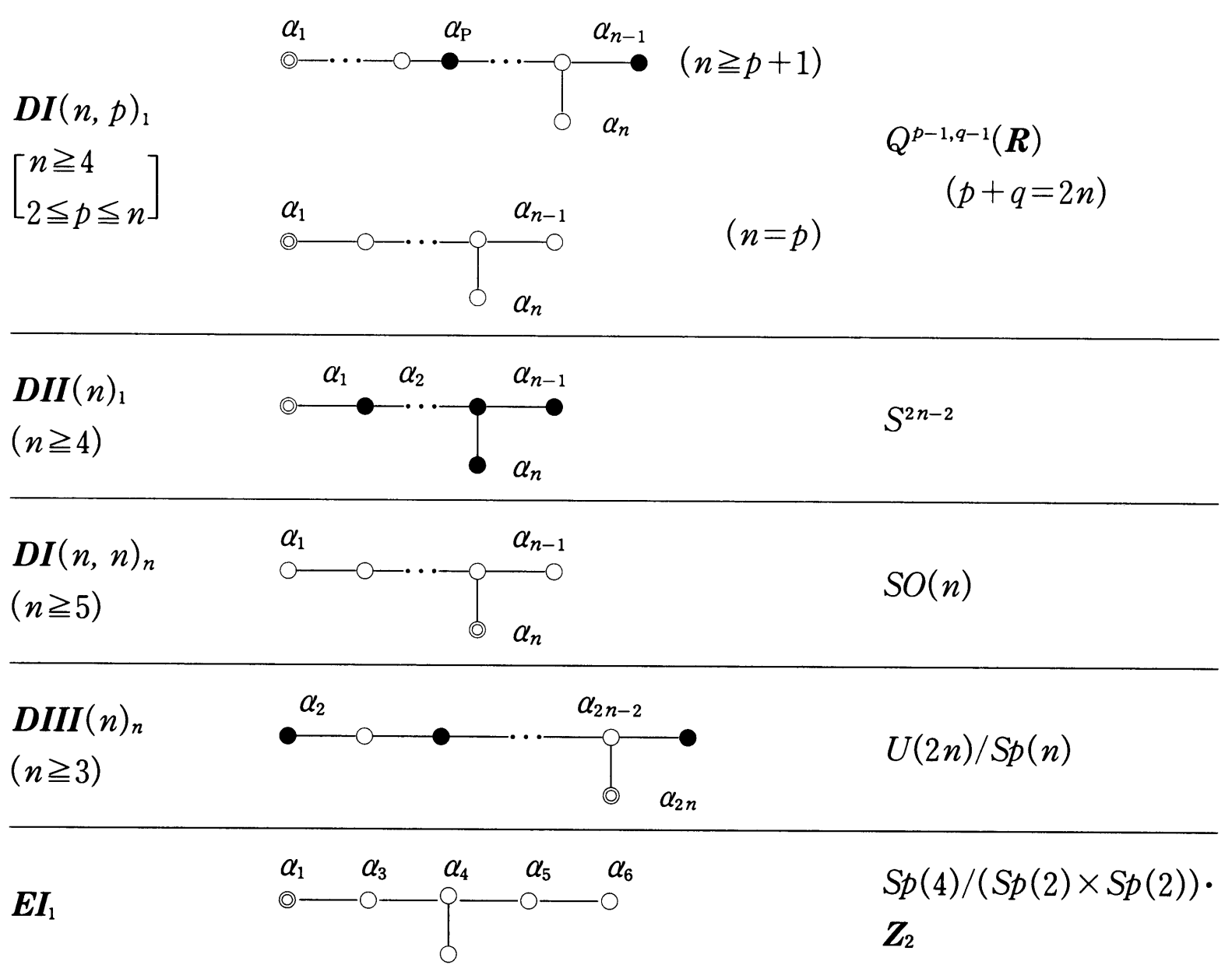


$E I V_{1}$

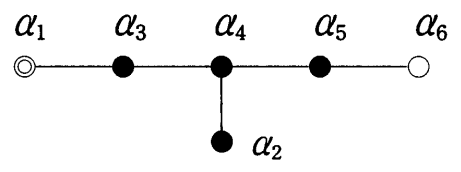

$$
P^{2}(\boldsymbol{C a y})
$$

$E V_{7}$

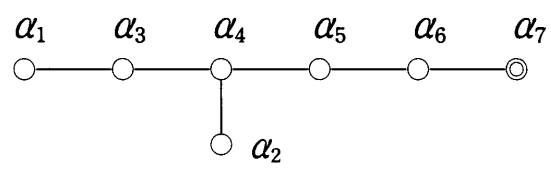

$$
S U(8) / S p(4) \cdot Z_{2}
$$

$\mathrm{EVII}_{3}$

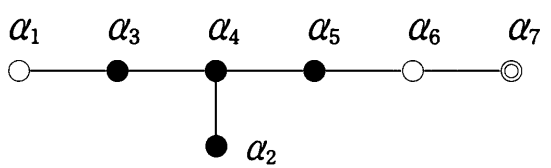

$$
E_{6} \cdot S O(2) / F_{4}
$$

\begin{tabular}{|c|c|c|c|}
\hline FSGLA & Dynkin diagram of $\Pi_{\Sigma}$ & & $m\left(\gamma_{i}\right)$ \\
\hline $\begin{array}{l}\boldsymbol{A I}(n)_{p} \\
{\left[\begin{array}{l}n \geqq 1 \\
1 \leqq p \leqq[(n+1) / 2]\end{array}\right]}\end{array}$ & 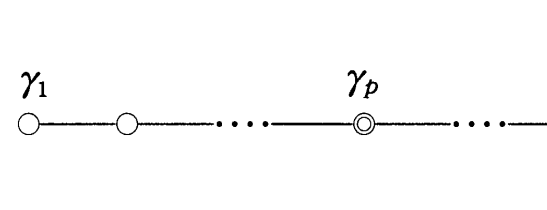 & $\begin{array}{r}\gamma_{n} \\
-0\end{array}$ & 1 \\
\hline $\begin{array}{l}\boldsymbol{A I I}(n)_{p} \\
{\left[\begin{array}{l}n \geqq 2 \\
1 \leqq p \leqq[n / 2]\end{array}\right]}\end{array}$ & 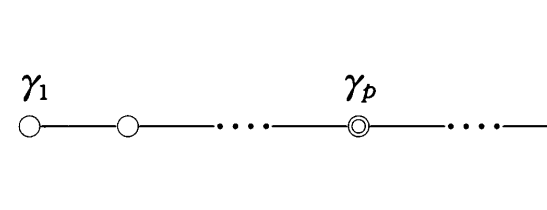 & $\gamma_{-\infty}^{\gamma_{n-1}}$ & 4 \\
\hline $\begin{array}{l}\boldsymbol{A I I I}(n, n)_{n} \\
(n \geqq 2)\end{array}$ & $\stackrel{\gamma_{1}}{\circ} \circ-\cdots-\cdots \stackrel{\gamma_{p}}{\circ}$ & & $\begin{array}{l}2(i<n) \\
1(i=n)\end{array}$ \\
\hline $\begin{array}{l}\boldsymbol{B I}(n, p)_{1} \\
{\left[\begin{array}{l}n \geqq 2 \\
2 \leqq p \leqq n\end{array}\right]}\end{array}$ & 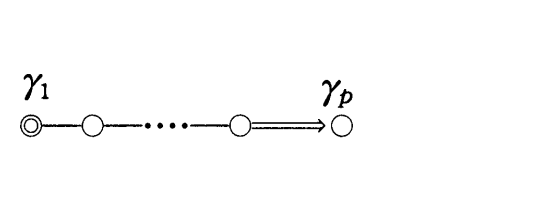 & & $\begin{array}{c}1 \quad(i<p) \\
2(n-p)+1 \quad(i=p)\end{array}$ \\
\hline $\begin{array}{l}\boldsymbol{B I I}(n)_{1} \\
(n \geqq 2)\end{array}$ & $\begin{array}{l}\gamma_{1} \\
\odot\end{array}$ & & $2 n-1$ \\
\hline $\begin{array}{l}\boldsymbol{C I}(n) \\
(n \geqq 3)\end{array}$ & 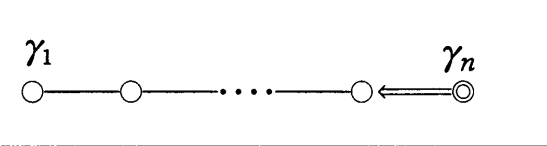 & & 1 \\
\hline $\begin{array}{l}\boldsymbol{C I I}(n, n)_{n} \\
(n \geqq 2)\end{array}$ & $\stackrel{\gamma_{1}}{\circ} \bigcirc \ldots \ldots$ & & $\begin{array}{l}4(i<\leqq n) \\
3(i=n)\end{array}$ \\
\hline
\end{tabular}

Appendix 2. Table II (Restricted root systems and multiplicties) 


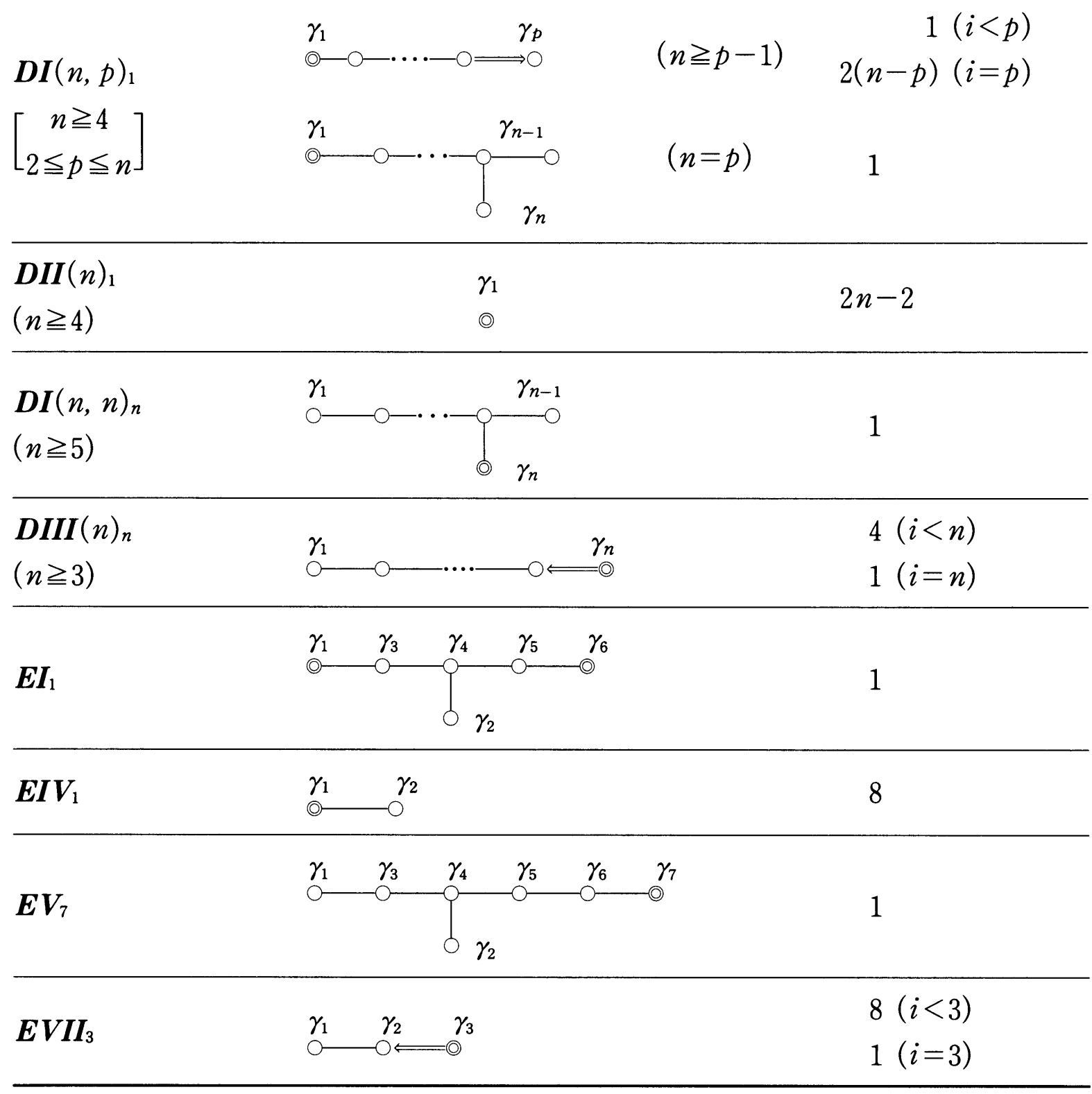

\section{References}

[AI] C. B. ALLENDOERFER, Rigidity for space of class greater than one, Amer. J. Math. 61 (1939), 633-644.

[Ar] S. ARAKI, On root systems and infinitesimal classification of irreducible symmetric spaces, J. Math. Osaka City Univ. 13(1962), 1-34.

[B] N. Bourbaki, Groupes et Algèbres de Lie, Chap 4, 5 et 6, Hermann, Paris, (1968).

[C] S. CoHN-Vossen, Zwei Sätzu über Starrheit der Eiflächen, Nacher. Ges. Wiss. Göttingen, 1(1927), 125-139.

[GQS]V. W. .GUILLEMIN, D. QUILLEN and S. STERNBERG, The classification of the irrducible complex algebras of infinite type, J. Analyse Math. 17(1967), 107-112. 
[H] S. HELEGASON, Differential Geometry, Lie Groups, and Symmetric Spaces, Academic Press, New York, 1978.

[Ka] E. KANEDA, Global rigidity of compact classical Lie groups, Hokkaido Math. J. 14 (1985), 365-397.

[KT] E. KANEDA and N. TANAKA, Rigidity for isometric imbeddings, J. Math. Kyoto Univ. 18(1978), 1-70.

[Ko] S. KOBAYASHI, Isometric imbeddings of compact symmetric spaces, Tohoku Math. J. 20(1967), 21-25.

[KN] S. KOBAYASHI and T. NAGANO, On filtered Lie algebras and geometric structures I, J. Math. Mech. 13(1964), 875-908 ; II, J. Math. Mech. 14(1965), 513-521 ; III, ibid., 679-706.

[N] L. NIRENBERG, The Weyl and Minkowski problems in the differential geometry in the large, Comm. Pure Appl. Math. 6(1953), 337-394.

[Tk] M. TAKEUCHI, Basic transformations of symmetric R-spaces, Osaka J. Math. 25(1988), 259-297.

[Tn] N. TANAKA, Rigidity for elliptic isometric imbeddings, Nagoya Math. J. 51(1973), 137 -160 .

Osaka University of Foreign Studies 8-1-1 Aomadani-Higashi

Mino'o, Osaka 562, Japan 